\title{
BMO-REGULARITY \\ IN LATTICES OF MEASURABLE FUNCTIONS ON SPACES OF HOMOGENEOUS TYPE
}

\author{
D. V. RUTSKY
}

\begin{abstract}
Let $X$ be a lattice of measurable functions on a space of homogeneous type $(S, \nu)$ (for example, $S=\mathbb{R}^{n}$ with Lebesgue measure). Suppose that $X$ has the Fatou property. Let $T$ be either a Calderón-Zygmund singular integral operator with a singularity nondegenerate in a certain sense, or the Hardy-Littlewood maximal operator. It is proved that $T$ is bounded on the lattice $\left(X^{\alpha} \mathrm{L}_{1}^{1-\alpha}\right)^{\beta}$ for some $\beta \in(0,1)$ and sufficiently small $\alpha \in(0,1)$ if and only if $X$ has the following simple property: for every $f \in X$ there exists a majorant $g \in X$ such that $\log g \in$ BMO with proper control on the norms. This property is called BMO-regularity. For the reader's convenience, a self-contained exposition of the BMO-regularity theory is developed in the new generality, as well as some refinements of the main results.
\end{abstract}

\section{$\S 0$. INTRODUCTION}

A quasi-Banach lattice $X$ of measurable functions on the unit circle $\mathbb{T}$ is said to be BMO-regula $\sqrt{1}$ if every $0 \neq f \in X$ admits a majorant $g, g \geq|f|$, such that $\|g\|_{X} \leq C\|f\|_{X}$ and $\|\log f\|_{\mathrm{BMO}(\mathbb{T})} \leq C$. This notion originates from the interpolation theory for analytic subspaces in such lattices. Under the condition that the lattice $X$ has some additional "natural" properties, $X$ gives rise to the space $X_{A}$ of all functions in $X$ that are boundary values of some functions of the Smirnov class. For instance, if $X=\mathrm{L}_{p}(\mathbb{T})$, then $X_{A}$ is simply the usual Hardy space $\mathrm{H}_{p}$.

It turns out (see [1]) that if some lattices $X$ and $Y$ are BMO-regular, then the real interpolation between $X_{A}$ and $Y_{A}$ is much similar to real interpolation between $X$ and $Y$, namely,

$$
\left(X_{A}, Y_{A}\right)_{\theta, p}=(X, Y)_{\theta, p} \cap\left(X_{A}+Y_{A}\right)
$$

(under some slight restirictions on the lattices). There are some indications that BMOregularity (or some similar property) is also necessary for this convenient relation (see [12, 13, 26]), but this remains an open problem to date.

The practical value of these results lies in the fact that BMO-regular lattices are surprisingly common. The following theorem illustrates this and also gives a clue how to find lattices that are not BMO-regular (the most convenient way is to find a lattice that does not satisfy condition 3 below). Recall that, by definition, the order dual $X^{\prime}$ of a lattice $X$ is the set of measurable functions $g$ such that $\int_{\mathbb{T}}|f g|<\infty$ for all $f$ in $X$. Suppose that the lattice $X$ is Banach and has the Fatou property (see $\$ 1$ for the definition). Suppose also that $0<\beta<1$.

2010 Mathematics Subject Classification. Primary 42B35; Secondary 42B20.

Key words and phrases. BMO-regularity, Muckenhoupt weights, singular integral operators, maximal function.

${ }^{1} \mathrm{BMO}$ stands for Bounded Mean Oscillation; see, e.g., 1 
Theorem A. The following statements are equivalent:

1) $X$ is BMO-regular;

2) $X^{\prime}$ is BMO-regular;

3) for all sufficiently small $\alpha, 0<\alpha<1$, the operator of harmonic conjugation is bounded on $\left(X^{\alpha} \mathrm{L}_{1}^{1-\alpha}\right)^{\beta}$.

Thus, the BMO-regularity of $X$ is closely related to a good behavior of the harmonic conjugation operator in certain lattices derived from $X$, which makes the class of BMOregular lattices interesting even beyond the scope of the interpolation theory of Hardytype spaces.

Theorem A is a deep result. For the first time it was obtained in [12] by using the Ky Fan-Kakutani fixed point theorem for multivalued mappings. Another ingredient which seemed essential at the time was complex analysis: although no spaces like $X_{A}$ appear in the statement of the theorem, the proof depended heavily on the use of such spaces along with a rather involved technique of the so-called analytic decomposition of unity subordinate to a given weight on the circle.

Among other things, in the present paper we prove Theorem A within the framework of real analysis only. The author has found this method when solving (and having solved successfully) a certain problem related to BMO-regularity and also originating from complex analysis; see [26]. The most important implication, however, is that the real methods make it possible to generalize this result from the case of the circle to other measurable spaces that admit a natural definition of BMO. We give a statement of this result for $\mathbb{R}^{n}$. As in the case of the circle, a lattice $X$ of measurable functions on $\mathbb{R}^{n}$ is said to be BMO-regular if for any $0 \neq f \in X$ there exists a majorant $g \geq|f|$ such that $\|g\|_{X} \leq C\|f\|_{X}$ and $\|\log g\|_{\text {BMO }} \leq C$. As before, we assume that $X$ has the Fatou property and $0<\beta<1$.

Theorem B. The following conditions are equivalent:

1) $X$ is BMO-regular;

2) $X^{\prime}$ is BMO-regular;

3) the Hardy-Littlewood maximal operator is bounded on $\left(X^{\alpha} \mathrm{L}_{1}^{1-\alpha}\right)^{\beta}$ for all suffciently small $\alpha, 0<\alpha<1$

4) all Calderón-Zygmund singular integral operators are bounded on $\left(X^{\alpha} \mathrm{L}_{1}^{1-\alpha}\right)^{\beta}$ for all sufficiently small $\alpha, 0<\alpha<1$;

5) one of the Riesz transformation

$$
R_{j} f(x) \stackrel{\text { def }}{=} \text { p.v. } \int_{\mathbb{R}^{n}} \frac{c_{n}\left(t_{j}-x_{j}\right)}{|t-x|^{n+1}} f(t) d t
$$

is bounded on $\left(X^{\alpha} \mathrm{L}_{1}^{1-\alpha}\right)^{\beta}$ for some $\alpha, 0<\alpha<1$.

Although in the case of $\mathbb{R}^{n}$ it is unclear whether BMO-regularity is somehow related to interpolation, theorem B shows that this property expresses in simple terms certain fundamental features of the lattice $X$, which makes the study of BMO-regularity justified in this general setting. The Ky Fan-Kakutani fixed point theorem still takes a major part in the proof. Moreover, in order to obtain the equivalence of statements 5 and 1 , we need the Grothendieck inequality. The use of this inequality in dealing with such problems, however, has been known for a long time. For the first time in a similar setting it was used by Rubio de Francia in [46]. We mention that in [12] the use of the Grothendieck inequality was avoided by complex methods, which are not available in the general setting.

The plan of the paper is as follows. In $\S \$ 1$ and 2 we give detailed statements of the results along with necessary definitions. It is often convenient to work with functions that 
depend on an additional variable beside $x \in \mathbb{R}^{n}$; furthermore, it is natural to consider an arbitrary measurable space of homogeneous type rather than $\mathbb{R}^{n}$. Since it is not possible to invoke the complex analysis techniques in this setting, instead we make use of the classes of Muckenhoupt weights $\mathrm{A}_{p}$ along with $\mathrm{BMO}$ and introduce certain properties similar to BMO-regularity but expressed in terms of these classes. In $₫ 3$ we give a number of known results to be used subsequently, and establish some auxiliary propositions. All simple claims of the present paper pertaining to BMO-regularity and $\mathrm{A}_{p}$-regularity are also verified in $\S ₫ 13$. The proofs are completed by two nontrivial arguments in $\S ₫ 4$ and 6. the first is based on the use of a fixed point theorem, the second depends on the Grothendieck theorem. In $\$ 5$ we give a version of the first argument for the case of couples of lattices and also establish the key points related to BMO-regularity for couples. Finally, in $₫ 7$ a more detailed historical review is given along with some further comments (it would have been inconvenient to do this before the whole theory has been laid out).

\section{§1. LATTICES AND BMO-REgULARITY}

In this section we introduce the basic notions and give a detailed exposition of BMOregularity. Suppose that $(S, \nu)$ is a space of homogeneous type, i.e., $S$ is a quasimetric space equipped with a Borel measure $\nu$ that has a doubling property: $\nu(B(x, 2 r)) \leq$ $c \nu(B(x, r))$ for all $x \in S$ and $0<r<\infty$, where $c$ is a constant called the doubling constant. Futher information on these spaces and real harmonic analysis on them can be found, e.g., in [8, 7]. It is usual in the literature on real harmonic analysis to consider only the typical case of $S$ being Euclidean spaces with Lebesgue measure; however, for the key results of this paper this restriction is not at all necessary. We are working with (real or complex) quasi-normed lattices of measurable functions on $(S \times \Omega, \nu \times \mu)$, where $(\Omega, \mu)$ is an arbitrary measurable space with a $\sigma$-finite measure $\mu$. The second variable $t \in \Omega$ does not usually cause much trouble in the arguments. Obviously, the case of one variable $x$ is included in this setting: we can take the point mass measure for $\mu$. By definition, a quasinormed lattice of measurable functions is a quasinormed space $X$ of measurable functions in which the norm is compatible with the natural order, that is, if $|f| \leq g$ a.e. for some function $g \in X$, then $f \in X$ and $\|f\|_{X} \leq C\|g\|_{X}$ for some constant $C$ independendent of $f$ and $g$. Usually, we have $C=1$; for example, it is always the case when the lattice has the Fatou property, which will be introduced shortly. Further information on lattices can be found, e.g., in [18, Chapter 10]. For simplicity we only work with lattices $X$ such that $\operatorname{supp} X=S \times \Omega$. Many interesting normed spaces are lattices; for example, so are the Lebesgue spaces $\mathrm{L}_{p}$, the Orlicz spaces $\mathrm{L}_{M}$, and the Lebesgue spaces $\mathrm{L}_{p(\cdot)}$ with variable exponent $p(\cdot)$, along with general modular spaces. The second variable makes it natural to work with $\mathrm{L}_{p}\left(\mathrm{~L}_{r}\right)$ and other lattices with mixed norm in this setting.

We list some key properties of quasinormed lattices and some related objects. For a Banach lattice $X$ of measurable functions, any order continuous functional $f$ on $X$ (order continuity means that, given a sequence $x_{n} \in X$ such that $\sup _{n}\left|x_{n}\right| \in X$ and $x_{n} \rightarrow 0$ a.e., we also have $f\left(x_{n}\right) \rightarrow 0$ ) has an integral representation $f(x)=\int x y_{f}$ for some function $y_{f}$ that is identified with $f$. The set $X^{\prime}$ of all such functionals is a Banach lattice with the norm defined by $\|f\|_{X^{\prime}}=\sup _{g \in X,\|g\|_{X}=1} \int|f g|$. The lattice $X^{\prime}$ is called the order dual of the lattice $X$. The norm of a lattice $X$ is said to be order continuous if for any nonincreasing sequence $x_{n} \in X$ converging to 0 a.e. we have $\left\|x_{n}\right\|_{X} \rightarrow 0$. The norm of a Banach lattice $X$ is order continuous if and only if $X^{*}=X^{\prime}$, i.e., if all norm continuous functionals on $X$ are order continuous. A lattice $X$ has the Fatou property if for any $f_{n}, f \in X$ such that $\left\|f_{n}\right\|_{X} \leq 1$ and the sequence $f_{n}$ converges to $f$ a.e. it is 
also true that $f \in X$ and $\|f\|_{X} \leq 1$. The Fatou property of a lattice $X$ is equivalent to the $(\nu \times \mu)$-closedness of the unit ball $B_{X}$ of the lattice $X$ (here and elsewhere by $(\nu \times \mu)$-convergence we mean convergence in measure on any measurable set $E$ such that $(\nu \times \mu)(E)<\infty)$. If the lattice $X$ is Banach, the Fatou property is equivalent to the order reflexivity of $X$, i.e., to the relation $X^{\prime \prime}=X$. Each of the above two properties is sufficient to guarantee that the lattice $X^{\prime}$ is a norming set of functionals for $X$, i.e., that $\|f\|_{X}=\sup _{g \in X^{\prime},\|g\|_{X^{\prime}}=1} \int f g$ for all $f \in X$. The order dual $X^{\prime}$ of a Banach lattice $X$ always has the Fatou property.

For any two quasinormed lattices $X$ and $Y$ on the same measurable space, the set of pointwise products of their functions

$$
X Y=\{f g \mid f \in X, g \in Y\}
$$

is a quasinormed lattice with the norm defined by

$$
\|h\|_{X Y}=\inf _{h=f g}\|f\|_{X}\|g\|_{Y} .
$$

If both lattices $X$ and $Y$ satisfy the Fatou property, then the lattice $X Y$ also has the Fatou property. This lattice multiplication is associative: if $X, Y$, and $Z$ are lattices of measurable functions on the same measurable space, then $(X Y) Z=X(Y Z)$ (here and elsewhere, if not stated otherwise, equality of lattices is understood as equality of sets coupled with equality of the quasinorms). By the well-known Lozanovskii factorization theorem (see [24]), for any Banach lattice $X$ satisfying the Fatou property the decomposition $X X^{\prime}=\mathrm{L}_{1}$ holds true. It is easily seen that if either of the lattices $X$ and $Y$ has order continuous quasinorm, then the norm of the lattice $X Y$ is also order continuous.

For any $\delta>0$ and a quasinormed lattice $X$, the lattice $X^{\delta}$ consists of all measurable functions $f$ having well-defined and finite quasinorms $\|f\|_{X^{\delta}}=\left\||f|^{1 / \delta}\right\|_{X}^{\delta}$. For example, $\mathrm{L}_{p}^{\delta}=\mathrm{L}_{\frac{p}{\delta}}$. For any product $X Y$ of quasinormed lattices and $\delta>0$ we have the natural relation $(X Y)^{\delta}=X^{\delta} Y^{\delta}$. If a lattice $X$ has the Fatou property, then the lattice $X^{\delta}$ also has the Fatou property; if the lattice $X$ has order continous quasinorm, then the lattice $X^{\delta}$ also has order continuous quasinorm. For any $0<\delta \leq 1$, if $X$ is a Banach lattice, then $X^{\delta}$ is also a Banach lattice. If $X$ and $Y$ are Banach lattices, then for any $0<\delta<1$ the lattice $X^{1-\delta} Y^{\delta}$ is also Banach; moreover, the following quite useful relation is true: $\left(X^{1-\delta} Y^{\delta}\right)^{\prime}=\left(X^{\prime}\right)^{1-\delta}\left(Y^{\prime}\right)^{\delta}$. For a lattice $X$ of measurable functions, by $X_{+}$we denote the cone of its positive functions $\{f \in X \mid f \geq 0$ a.e. $\}$.

For a quasinormed lattice $X$ and a function $w$ positive a.e., the weighted lattice $X(w)$ is defined as the set $X(w)=\{w f \mid f \in X\}$ with the quasinorm $\|f\|_{X(w)}=$ $\left\|f w^{-1}\right\|_{X}$. For example, $\mathrm{L}_{\infty}(w)=\{f|| f \mid \leq C w$ a. e. $\}$. It is easily seen that $[X(w)]^{\prime}=$ $X^{\prime}\left(w^{-1}\right)$. Notice that this definition of the weighted Lebesgue space $\mathrm{L}_{p}(w)$ differs from the "classical" one, with the norm defined as $\|f\|_{p, w}^{p}=\int|f|^{p} w$, which is often found in the literature; the latter norm corresponds to the norm of the lattice $\mathrm{L}_{p}\left(w^{-\frac{1}{p}}\right)$ in our notation. Thus, all weighted lattices are defined in a unified way everywhere in

\footnotetext{
${ }^{2}$ Note added in translation. It was brought to the author's attention that this definition does not work well for weights $w$ such that $w=+\infty$ on a set of positive measure, and that weighted spaces with such weights do appear in some arguments in $\$ 3$ For our purposes, a correct definition extending $X(w)$ to such weights is slightly different: we define the set by $X(w)=\left\{g \mid \frac{g}{w} \in X\right\}$ for weights $w$ such that $0 \leq w \leq \infty$ almost everywhere. Thus, $g=0$ on the set where $w=0$, the restriction of $g$ to the set $\{w=+\infty\}$ is an arbitrary measurable function, and $\|\cdot\|_{X(w)}$ is a quasiseminorm for weights $w$ such that the set $\{w=+\infty\}$ has positive measure. In any case, if $w \in\{0,+\infty\}$ on a set of positive measure, we regard $X(w)$ as merely a set of functions with a seminorm under our conventions, because then $\operatorname{supp} X(w) \neq \operatorname{supp} X$. Note that it is usually possible to avoid dealing with such "bad" weights; see Proposition 14 below.
} 
this paper; however, attention must be paid to this difference. We adopt the natural conventions $0^{-1}=\infty$ and $\infty^{-1}=0$ in all expressions involving weights.

The (centered) Hardy-Littlewood maximal operator

$$
M f(x, t)=\sup _{r>0} \frac{1}{\nu(B(x, r))} \int_{B(x, r)}|f(z, t)| d \nu(z) \quad x \in S, \quad t \in \Omega,
$$

and the Fefferman-Stein maximal operator

$$
M^{\sharp} f(x, t)=\sup _{r>0} \frac{1}{\nu(B(x, r))} \int_{B(x, r)}\left|f(z, t)-\frac{1}{\nu(B(x, r))} \int_{B(x, r)} f(a, t) d \nu(a)\right| d \nu(z)
$$

are well defined for a.e. $x \in S$ and $t \in \Omega$, and for all measurable functions $f$ on $(S \times \Omega, \nu \times \mu)$ that are locally integrable in the first variable. For convenience, we assume that $\log 0=-\infty$ and $M^{\sharp}(-\infty)=0$. We say that a nonnegative measurable function $w$ on $(S \times \Omega, \nu \times \mu)$ belongs to the Muckenhoupt class $\mathrm{A}_{p}$ for some $1 \leq p<\infty$ with a constant $C$ if

$$
\underset{t \in \Omega}{\operatorname{ess} \sup }\|M\|_{\mathrm{L}_{p}\left(w^{-1 / p}(\cdot, t)\right) \rightarrow \mathrm{L}_{p, \infty}\left(w^{-1 / p}(\cdot, t)\right)} \leq C .
$$

For $p>1$, this condition is equivalent to $\operatorname{ess}^{\sup _{t \in \Omega}}\|M\|_{\mathrm{L}_{p}\left(w^{-1 / p}(\cdot, t)\right)} \leq C^{\prime}$ with $C^{\prime}$ equivalent to $C$ up to some multiplicative constant. The class $A_{1}$ is characterized by the estimate $M w \leq C^{\prime} w$ a.e. (this will be used in the arguments), while the classes $\mathrm{A}_{p}$ for $p>1$ are characterized by the well-known Muckenhoupt condition

$\underset{x \in S, t \in \Omega}{\operatorname{ess} \sup _{r>0}}\left[\frac{1}{\nu(B(x, r))} \int_{B(x, r)} w(u, t) d \nu(u)\right]\left[\frac{1}{\nu(B(x, r))} \int_{B(x, r)} w(u, t)^{-\frac{1}{p-1}} d \nu(u)\right]^{p-1}<\infty$

(we do not use this in the paper); see, e.g., 7]. We say that a measurable function $f$ on $(S \times \Omega, \nu \times \mu)$ belongs to BMO with a constant $C$ if $\left\|M^{\sharp} f\right\|_{L_{\infty}} \leq C$. The Muckenhoupt classes and BMO are studied fairly well, and a great number of useful equivalent definitions are known (see, e.g., [42, 7]). However, we only require the definitions stated above and a small number of well-known properties, which we shall introduce as the need arises. It is well known (see 7, Chapter 5, 6.2]) that the relation $w \in \mathrm{A}_{p}$ implies $\log w \in \mathrm{BMO}$ and, vice versa, if $\log w \in \mathrm{BMO}$, then $w^{\delta} \in \mathrm{A}_{p}$ for sufficiently small $\delta$, $0<\delta<\delta_{0}$. The value of $\delta_{0}$ depends only on the BMO constant of the function log $w$ and on $1<p<\infty$, with an appropriate estimate (i.e., the BMO constant of $\log w$ admits an estimate in terms of $p$ and the $\mathrm{A}_{p}$ constant of the weight $w$, and the $\mathrm{A}_{p}$-constant of $w^{\delta}$ can likewise be estimated in terms of $p, \delta$, and the BMO constant of the function $\log w)$. If $w \in \mathrm{A}_{p}$ and $1 \leq p<\infty$, then also $w \in \mathrm{A}_{q}$ for all $q>p$ with an estimate for the $\mathrm{A}_{q}$ constant in terms of the $\mathrm{A}_{p}$ constant. Any weight $w \in \mathrm{A}_{p}, 1<p<\infty$, also belongs to $\mathrm{A}_{q}$ for some $1<q<p$, with an estimate for the constant.

Definition 1. A quasinormed lattice $X$ on $(S \times \Omega, \nu \times \mu)$ is $\mathrm{A}_{p}$-regular with constants $(C, m)$ if for any $f \in X$ there exists a majorant $g \in X, g \geq|f|$ such that $\|g\|_{X} \leq m\|f\|_{X}$ and $g \in \mathrm{A}_{p}$ with constant $C$. A quasinormed lattice $X$ is BMO-regular with constants $(C, m)$ if for any $f \in X$ there exists a majorant $g \in X, g \geq|f|$ such that $\|g\|_{X} \leq m\|f\|_{X}$ and $\log g \in \mathrm{BMO}$ with constant $C$. This function $g$ is called an $\mathrm{A}_{p}$-majorant of a BMOmajorant for $f$.

The $\mathrm{A}_{p}$-regularity property plays an auxiliary technical role as far as the main results of this paper are concerned. It is convenient that the set of $\mathrm{A}_{p}$-majorants for a given function $f$ has nice properties (see Proposition 16 below). However, $\mathrm{A}_{p}$-regularity may be interesting in its own right. It is easily seen that $\mathrm{A}_{p}$-regularity implies $\mathrm{A}_{q}$-regularity for all $q>p$, as well as BMO-regularity. Moreover, $\mathrm{A}_{p}$-regularity implies $\mathrm{A}_{q}$-regularity for some $1<q<p$ if $p>1$. On the other hand, if a lattice $X$ is BMO-regular, then for 
any $1<p<\infty$ the lattice $X^{\delta}$ is $\mathrm{A}_{p}$-regular for all sufficiently small $\delta>0$. Thus, we see that $\mathrm{A}_{p}$-regularity is a refinement of BMO-regularity. The same ideas can be used to define the BMO norm by

$$
\|f\|_{\mathrm{BMO}}=\inf \left\{q>0 \mid e^{\frac{f}{q}} \in \mathrm{A}_{2} \text { with a constant } c_{0}>1\right\},
$$

as it was done, e.g., in [26]; we omit the details here.

$\mathrm{A}_{1}$-regularity admits the following characterization.

Proposition 1. A quasinormed lattice $X$ on $(S \times \Omega, \nu \times \mu)$ is $\mathrm{A}_{1}$-regular if and only if the maximal operator $M$ is bounded on $X$.

For the proof, it suffices to observe that any $\mathrm{A}_{1}$-majorant $g$ satisfies $M g \leq C g$. On the other hand, if the maximal operator $M$ is bounded on $X$ with norm $C$, then for any $f \in X$ we can construct inductively a function $g=\sum_{n \geq 0} c^{-n-1}(2 C)^{-n} f_{n}, f_{0}=|f|$, $f_{n+1}=M f_{n}$, where $c$ is the triangle inequality constant for the quasinorm of $X$. The function $g$ is a suitable $\mathrm{A}_{1}$-majorant for $f$, because $\left\|f_{n}\right\|_{X} \leq C^{n}\|f\|_{X},\|g\|_{X} \leq 2 c\|f\|_{X}$ and $M g \leq 2 C c g$.

A quasinormed lattice $X$ on $(\Sigma, \sigma)$ is said to be rearrangement invariant (or symmetric) if for any $f \in X$ and any bijection $\tau: \Sigma \rightarrow \Sigma$ preserving the measure $\sigma$ we have $\|f \circ \tau\|_{X}=\|f\|_{X}$. We use the standard composition notation $(f \circ \tau)(x)=f(\tau(x))$ for $x \in \Sigma$. It is well known that any rearrangement invariant Banach lattice $X$ on a measurable space with $\sigma$-finite measure such that $X$ is an intermediate space between $\mathrm{L}_{1}$ and $\mathrm{L}_{\infty}$ (i.e., $\mathrm{L}_{1} \cap \mathrm{L}_{\infty} \subset X \subset \mathrm{L}_{1}+\mathrm{L}_{\infty}$ ) is an interpolation space between $\mathrm{L}_{1}$ and $\mathrm{L}_{\infty}$ for the class of quasilinear operators (see, e.g., 9, II, Theorem 2.a.10] and [15]). If we take $(\Sigma, \sigma)=(S, \nu) \times(\Omega, \mu)$, the quasilinear operator $M^{\delta}(f)=\left(M|f|^{\delta}\right)^{\frac{1}{\delta}}$ is bounded on any such lattice for any $0<\delta<1$. It follows at once that $M$ is bounded on $X^{\delta}$. Combining this fact with Proposition 1 yields the following proposition.

Proposition 2. Any rearrangement invariant quasi-Banach lattice $X$ on $(S \times \Omega, \nu \times \mu)$ such that $X^{p}$ is an intermediate Banach space for the couple $\left(\mathrm{L}_{1}, \mathrm{~L}_{\infty}\right)$ for some $p>0$ is $\mathrm{BMO}$-regular, and the lattice $X^{q}$ is $\mathrm{A}_{1}$-regular for $0<q<p$.

It is not clear if such a result holds true for lattices on $(S \times \Omega, \nu \times \mu)$ that are rearrangement invariant in the first variable only; for $\mathrm{A}_{1}$-regularity this is not the case (see Proposition 8 below). We point out another necessary condition that follows at once from the fact that an $\mathrm{A}_{p}$ weight is locally $\mathrm{L}_{r}$-integrable for some $r>1$.

Proposition 3. Suppose that the measurable space $(S, \nu)$ has the following property: $\nu(B(x, 2 r) \backslash B(x, r))>0$ for some $x \in S$ and all sufficiently small $r$. Then the lattice $\mathrm{L}_{q}$ is not $\mathrm{A}_{p}$-regular for all $1 \leq p<\infty$ and $0<q \leq 1$.

Indeed, under the conditions of Proposition 3 we can find a monotone decreasing sequence $r_{n}>0, n \in \mathbb{N}$, such that $2 r_{n+1}<r_{n}$ for all $n$ and $0<a_{n}=\nu\left(B\left(x, 2 r_{n}\right) \backslash\right.$ $\left.B\left(x, r_{n}\right)\right)<2^{-n}$. Take $f=\sum_{n} \frac{n^{-2}}{a_{n}} \chi_{B\left(x, 2 r_{n}\right) \backslash B\left(x, r_{n}\right)}$. It is easily seen that $f \in \mathrm{L}_{q}$ for $0<q \leq 1$. Suppose that $f$ has an $\mathrm{A}_{p}$-majorant $g$. Then by the properties of $\mathrm{A}_{p}$ weights we have $g \chi_{B\left(x, r_{n}\right)} \in \mathrm{L}_{s}$ for some $s>1$ and $n$, whence $f \chi_{B\left(x, r_{n}\right)} \in \mathrm{L}_{s}$. However,

$$
\int_{B\left(x, r_{n}\right)}|f|^{s}=\sum_{k \geq n} k^{-2 s} a_{k}^{1-s} \geq \sum_{k \geq n} k^{-2 s} 2^{k(s-1)}=\infty,
$$

a contradiction; this proves Proposition 3 .

Thus, we see that BMO-regularity occurs fairly often and is a rather convenient property in relation to the algebraic structure of the class of lattices. It is easy to show that if $X$ is a BMO-regular lattice, then $X^{\alpha}$ is also BMO-regular for all $\alpha>0$. If $X$ 
and $Y$ are BMO-regular, then $X Y$ is also BMO-regular. It turns out that, under the Fatou property, BMO-regularity is also stable with respect to duality and division by a BMO-regular lattice.

Theorem 1. Suppose that $X$ is a Banach lattice with the Fatou property on $(S \times \Omega, \nu \times \mu)$. Fix $0<\beta<1$. The following conditions are equivalent.

1) $X$ is BMO-regular.

2) $X \mathrm{~L}_{q}$ is BMO-regular for some $0<q<\infty$.

3) The maximal operator $M$ is bounded on $\left(X^{\alpha} \mathrm{L}_{1}^{1-\alpha}\right)^{\beta}$ for all sufficiently small $0<\alpha \leq 1$.

4) $M$ is bounded in $\left(X^{\alpha} \mathrm{L}_{1}^{1-\alpha}\right)^{\beta}$ for some $0<\alpha \leq 1$.

5) $X^{\prime}$ is BMO-regular.

The only really difficult implication in this theorem is 2 ) $\Rightarrow 1$ ) ("division by $\mathrm{L}_{q}$ "). The following is a more detailed statement of this implication.

Theorem 2. Suppose that $X$ is a Banach lattice with the Fatou property on $(S \times \Omega, \nu \times \mu)$. Suppose also that, for some $1<q<\infty, X \mathrm{~L}_{q}$ is a Banach lattice that is $\mathrm{A}_{p}$-regular for some $1 \leq p<\infty$. Then $X$ is $\mathrm{A}_{p+1}$-regular.

The lattices $X=\mathrm{L}_{\infty}\left(\ell^{p}\right)$ show (see Proposition 10 below) that, at least in the typical case of the space $\mathbb{R}^{n}$ with Lebesgue measure, the $\mathrm{A}_{1}$-regularity of the lattice $X \mathrm{~L}_{q}$ does not imply the $\mathrm{A}_{1}$-regularity of the lattice $X$, so that, as stated, Theorem 2 cannot be improved to show that $X$ is $\mathrm{A}_{p}$-regular, at least if $p=1$. The proof of Theorem 2 is rather involved and requires some preparations. Since this proof does not depend on $\S \S 02$ in any way, we postpone it until 4 for convenience. Moreover, in \$5 we give another proof of Theorem 2, which is based on a similar $\mathrm{A}_{p}$-regularity property for couples of lattices.

So, we take Theorem 2 for granted for the time being and proceed to the proof of Theorem 1. If a lattice $X$ satisfies condition 2) of Theorem 1, then the lattice $X^{\delta}$ satisfies the assumptions of Theorem 2 for a certain sufficiently small $\delta>0$ (and for another $q$ ); therefore, this lattice is BMO-regular (and condition 1 is satisfied). Let $p=\frac{1}{\beta}$. In order to verify the implication 5) $\Rightarrow 3$ ) in Theorem 1 we notice that, under condition 5), for all sufficiently small $\alpha>0$ the lattice $X^{\prime \alpha}$ is $\mathrm{A}_{p}$-regular. This means that for any $h \in X^{\prime \alpha}$ there exists an $\mathrm{A}_{p}$-majorant $g_{h} \in X^{\prime \alpha}, g_{h} \in \mathrm{A}_{p}$, with suitable $\mathrm{A}_{p}$ constant and $X^{\prime \alpha}$ norm estimates. These estimates allow us to obtain the following inequality for arbitrary $f \in X^{\alpha} \mathrm{L}_{1}^{1-\alpha}=\left(X^{\alpha}\right)^{\prime}$ :

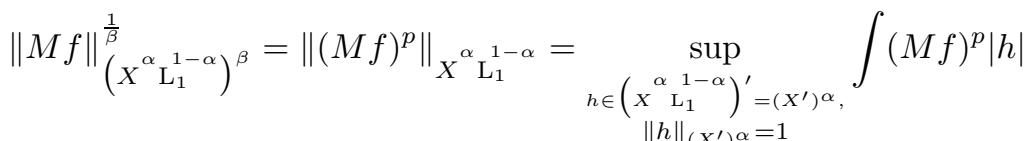

$$
\begin{aligned}
& \leq \sup _{h \in\left(X^{\prime}\right)^{\alpha},\|h\|_{\left(X^{\prime}\right)^{\alpha}=1}} \int(M f)^{p} g_{h} \leq c \sup _{h \in\left(X^{\prime}\right)^{\alpha},\|h\|_{\left(X^{\prime}\right)^{\alpha}=1}} \int|f|^{p} g_{h} \\
& \leq c \sup _{h \in\left(X^{\prime}\right)^{\alpha},\|h\|_{\left(X^{\prime}\right)^{\alpha}=1}}\left\||f|^{p}\right\|_{X^{\alpha} L_{1}^{1-\alpha}}\left\|g_{h}\right\|_{\left(X^{\prime}\right)^{\alpha}} \\
& \leq c^{\prime}\left\||f|^{p}\right\|_{X^{\alpha} L_{1}^{1-\alpha}}=c^{\prime}\|f\|_{\left(X^{\alpha} L_{1}^{1-\alpha}\right)^{\beta}}^{\frac{1}{\beta}}
\end{aligned}
$$

with some constants $c$ and $c^{\prime}$ independent of $f$, which proves the implication 5) $\Rightarrow 3$ ). The implication 3$) \Rightarrow 4$ ) is trivial. Finally, in order to verify that 4$) \Rightarrow 2$ ), we observe that under condition 4) the lattice $\left(X^{\alpha} \mathrm{L}_{1}^{1-\alpha}\right)^{\beta}$ is $\mathrm{A}_{1}$-regular by Proposition 1 so that the lattice

$$
\left[\left(X^{\alpha} \mathrm{L}_{1}^{1-\alpha}\right)^{\beta}\right]^{\frac{1}{\alpha \beta}}=X \mathrm{~L}_{1}^{\frac{1-\alpha}{\alpha}}=X \mathrm{~L}_{\frac{\alpha}{1-\alpha}}
$$


is BMO-regular. Thus $X$ satisfies condition 2) for $q=\frac{\alpha}{1-\alpha}$. We have established the implications 5) $\Rightarrow 3) \Rightarrow 4) \Rightarrow 2) \Rightarrow 1$ ). The implication 1) $\Rightarrow 5$ ) for a lattice $X$ is equivalent to the already established implication 5) $\Rightarrow 1$ ) for the lattice $X^{\prime}$, because the Fatou property ensures that $X=X^{\prime \prime}$. This concludes the proof of Theorem 1.

The proof of the implication 5) $\Rightarrow 3$ ) in Theorem 1 and Proposition 1 yield the following refinement of the self-duality property.

Proposition 4. Suppose that $X$ is a Banach lattice on $(S \times \Omega, \nu \times \mu)$ such that $X^{\prime}$ is a norming space for $X$. If $X^{\prime}$ is $\mathrm{A}_{1}$-regular, then $X^{\frac{1}{q}}$ is $\mathrm{A}_{1}$-regular for $q>1$. If $X^{\prime}$ is $\mathrm{A}_{p}$-regular for some $p>1$, then $X^{\frac{1}{q}}$ is $\mathrm{A}_{1}$-regular for some $q<p$ (which means that $X^{\frac{1}{r}}$ is $\mathrm{A}_{1}$-regular for all $r \geq q$ ).

It suffices to carry out a suitable estimate similar to (1) for every case of this proposition. If $X^{\prime}$ is $\mathrm{A}_{p}$-regular for some $p>1$, then $X^{\prime}$ is also $\mathrm{A}_{q}$-regular for some $1<q<p$. If $X^{\prime}$ is $\mathrm{A}_{1}$-regular, then $X^{\prime}$ is also $\mathrm{A}_{q}$-regular for all $q>1$. The assumptions imply that for any $h \in X^{\prime}$ there exists a suitable $\mathrm{A}_{q}$-majorant $g_{h}$. Then

$$
\begin{aligned}
\|M f\|_{X^{\frac{1}{q}}}^{q}=\left\|(M f)^{q}\right\|_{X} & =\sup _{\substack{h \in X^{\prime},\|h\|_{X^{\prime}}=1}} \int(M f)^{q} h \\
& \leq \sup _{\substack{h \in X^{\prime},\|h\|_{X^{\prime}}=1}} \int(M f)^{q} g_{h} \leq c \sup _{\substack{h \in X^{\prime},\|h\|_{X^{\prime}}=1}} \int|f|^{q} g_{h} \\
& \leq c \sup _{\substack{h \in X^{\prime},\|h\|_{X^{\prime}}=1}}\left\||f|^{q}\right\|_{X}\left\|g_{h}\right\|_{X^{\prime}} \leq c^{\prime}\|f\|_{X^{\frac{1}{q}}}^{q},
\end{aligned}
$$

and the $\mathrm{A}_{1}$-regularity of $X^{\frac{1}{q}}$ follows by Proposition 1

Generally speaking, the $\mathrm{A}_{p}$-regularity property is not self-dual, because for the spaces $\mathbb{R}^{n}$ with Lebesgue measure the lattice $\mathrm{L}_{\infty}$ is $\mathrm{A}_{1}$-regular, but its order dual $\mathrm{L}_{1}$ is not $\mathrm{A}_{p}$-regular for all $1 \leq p<\infty$ by Proposition 3 .

Theorem 1 easily yields a general statement about "divisibility" of BMO-regularity, as well as a number of useful properties.

Proposition 5. Suppose that $X$ and $Y$ are Banach lattices with the Fatou property on $(S \times \Omega, \nu \times \mu)$. If both $X Y$ and $Y$ are BMO-regular, then $X$ is also BMO-regular. In particular, if the lattices $Y$ and $Y(w)$ are both BMO-regular for some weight $w$, then $\|\log w(\cdot, \omega)\|_{\mathrm{BMO}} \leq C$ for a.e. $\omega \in \Omega$ and some constant $C$.

Indeed, since under these assumptions the lattice $(X Y) Y^{\prime}=X\left(Y Y^{\prime}\right)=X \mathrm{~L}_{1}$ is BMOregular, Theorem 1 1 shows that the lattice $X$ is also BMO-regular. Furthermore, $Y(w)=$ $Y \mathrm{~L}_{\infty}(w)$. If $Y$ and $Y(w)$ are BMO-regular, then $\mathrm{L}_{\infty}(w)$ is also BMO-regular, which yields a BMO-estimate for $\log w$ at once.

Proposition 6. Suppose that $X$ and $Y$ are some BMO-regular quasinormed lattices on $(S \times \Omega, \nu \times \mu)$. Then the lattices $X+Y$ and $X \cap Y$ are also BMO-regular.

It suffices to notice that for $f \in X \cap Y$ and the corresponding BMO-majorants $g \in X$ and $h \in Y$ the function $g \wedge h$ is a BMO-majorant for $f$ in $X \cap Y$. If $f=g+h \in$ $X+Y,\|g\|_{X}+\|h\|_{Y} \leq c\|f\|_{X+Y}$, and $G, H$ are some BMO-majorants in $X, Y$ for $g, h$, respectively, then $2(G \vee H)$ is a BMO-majorant for $f$ in $X+Y$, because $2(G \vee H) \geq g+h$ and $\|G \vee H\|_{X+Y} \leq\|G\|_{X}+\|H\|_{Y} \leq C\|f\|_{X+Y}$. It is remarkable that these properties follow easily from condition 4) of Theorem 1 and relations $(X \cap Y) Z=X Z \cap Y Z$, $(X+Y) Z=X Z+Y Z$ for arbitrary lattices (in the sense of equivalence of norms), provided that $X$ and $Y$ satisfy the assumptions of Theorem 1, 
We mention yet another interesting application of duality. Recall that, for a quasinormed lattice $X$ on $(S \times \Omega, \nu \times \mu)$ and a quasinormed lattice $\Xi$ on a $\sigma$-finite measurable space $\left(\Omega_{1}, \mu_{1}\right)$, the lattice $X(\Xi)$ is defined as a maximal linear set of measurable functions on $\left(S \times \Omega \times \Omega_{1}, \nu \times \mu \times \mu_{1}\right)$ such that the norm $\|f\|_{X(\Xi)}=\|g\|_{X}, \quad g(t, \omega)=\|f(t, \omega, \cdot)\|_{\Xi}$, is well defined and finite. Working with such lattices sometimes poses certain difficulties related to measurability. If $X$ and $\Xi$ are Banach lattices on $(S \times \Omega, \nu \times \mu)$ and $\left(\Omega_{1}, \mu_{1}\right)$, respectively, then the natural formula $[X(\Xi)]^{\prime}=X^{\prime}\left(\Xi^{\prime}\right)$ holds true. Suppose that $X$ and $Y$ are quasinormed lattices on $(S \times \Omega, \nu \times \mu), \Phi$ and $\Psi$ are quasinormed lattices on $\left(\Omega_{1}, \mu_{1}\right)$. If the lattice $X(\Psi) Y(\Phi)$ has the Fatou property and $\Psi \Phi$ is a separable space, then, by [26, Proposition 18], we have the natural relation $X(\Psi) Y(\Phi)=(X Y)(\Psi \Phi)$. The following simple observation prepares for Proposition 8 below.

Proposition 7. If a quasinormed lattice $X$ on $(S \times \Omega, \nu \times \mu)$ is BMO-regular, then the lattice $X\left(\mathrm{~L}_{\infty}\right)$ on $\left(S \times \Omega \times \Omega_{1}, \nu \times \mu \times \mu_{1}\right)$ is also BMO-regular with the same constants. The same is true for $\mathrm{A}_{p}$-regularity.

For the proof, it suffices to observe that $f \in X\left(\mathrm{~L}_{\infty}\right)$ means by definition that the function $g(x, t)=\operatorname{ess}_{\sup _{\omega \in \Omega_{1}}}|f(x, t, \omega)|, x \in S, t \in \Omega$, belongs to $X$ with a suitable estimate of its quasinorm. If $h$ is a suitable majorant for $f$ in $X$, then $\tilde{h}(x, t, \omega)=h(x, t)$, $x \in S, t \in \Omega, \omega \in \Omega_{1}$ is a suitable majorant for $f$ in $X\left(\mathrm{~L}_{\infty}\right)$.

Proposition 8. Suppose that $X$ is a BMO-regular quasinormed lattice on $(S \times \Omega, \nu \times \mu)$. Then the lattice $X\left(\mathrm{~L}_{p}\right)$ on $\left(S \times \Omega \times \Omega_{1}, \nu \times \mu \times \mu_{1}\right)$ is BMO-regular for all $0<p \leq \infty$.

Indeed, the lattice $\mathrm{L}_{1}\left(\mathrm{~L}_{\infty}\right)$ is BMO-regular by Proposition 7 , therefore, $\mathrm{L}_{\infty}\left(\mathrm{L}_{1}\right)=$ $\left[\mathrm{L}_{1}\left(\mathrm{~L}_{\infty}\right)\right]^{\prime}$ is also BMO-regular. Thus, $\mathrm{L}_{\infty}\left(\mathrm{L}_{p}\right)$ is BMO-regular for all $0<p \leq \infty$. Multiplying it by the lattice $X\left(\mathrm{~L}_{\infty}\right)$, which is BMO-regular by Proposition 7 , we arrive at the BMO-regularity of the lattice $X\left(\mathrm{~L}_{p}\right)$, as claimed.

The BMO-regularity property of the lattice $\mathrm{L}_{\infty}\left(\ell^{p}\right)$ admits certain refinements, which we are going to discuss in the next lines. Since the maximal operator $M$ is bounded on $X=\mathrm{L}_{q}\left(\ell^{p}\right)$ for all $1<p, q<\infty$ (see, e.g., [29]), $X$ is $\mathrm{A}_{1}$-regular by Proposition 1 , Furthermore, since $\mathrm{L}_{q}\left(\mathrm{~L}_{p}\right)=\mathrm{L}_{\infty}\left(\mathrm{L}_{r}\right) \mathrm{L}_{q}\left(\mathrm{~L}_{q}\right)$ if $q>p$ and $\frac{1}{r}=\frac{1}{p}-\frac{1}{q}$, Theorem 2 yields the $\mathrm{A}_{2}$-regularity of the lattice $\mathrm{L}_{\infty}\left(\mathrm{L}_{r}\right)$; the exponent $r$ takes all values $1<r<\infty$. Observe that, by [7, 6.1, Chapter 5], the $\mathrm{A}_{p}$-regularity property improves in a certain way as we raise the lattice to a small power.

Proposition 9. Let $X$ be an $\mathrm{A}_{p}$-regular quasinormed lattice on $(S \times \Omega, \nu \times \mu)$. Then $X^{\theta}$ is $\mathrm{A}_{1+\theta(p-1)}$-regular for all $0<\theta \leq 1$.

This proposition coupled with the established $\mathrm{A}_{2}$-regularity of $\mathrm{L}_{\infty}\left(\mathrm{L}_{r}\right)$ implies that the lattice $\mathrm{L}_{\infty}\left(\mathrm{L}_{s}\right)=\left[\mathrm{L}_{\infty}\left(\mathrm{L}_{r}\right)\right]^{\frac{r}{s}}$ is $\mathrm{A}_{1+\frac{r}{s}}$-regular for all $1<r<s<\infty$.

On the other hand, at least in the case of lattices on $\mathbb{R}^{n}$, the maximal operator $M$ is not bounded on $\mathrm{L}_{\infty}\left(\ell^{p}\right)$ for $p<\infty$ (see, e.g., [7, Chapter 2]), whence $\mathrm{L}_{\infty}\left(\ell^{p}\right)$ is not $\mathrm{A}_{1}$-regular if $p<\infty$. We show that in the case of $\mathbb{R}^{n}$ the lattice $\mathrm{L}_{\infty}\left(\ell^{1}\right)$ is not $\mathrm{A}_{p^{-}}$ regular for all $1<p<\infty$. Suppose that, to the contrary, this lattice is $\mathrm{A}_{p}$-regular for some $1<p<\infty$. By the proof of the implication 5) $\Rightarrow 3$ ) in Theorem 1 (see also Proposition 13 in the next section), the Riesz transformation $R=R_{1}$ (or the Hilbert transformation $R=H$ if $n=1)$ is then bounded on $\left[\mathrm{L}_{\infty}\left(\ell^{1}\right)\right]^{\prime \frac{1}{p}}=\mathrm{L}_{p}\left(\ell^{\infty}\right)$, which cannot be true. For example, take $f(x)=\left\{g\left(x+x_{j}\right)\right\}_{j \in \mathbb{N}} \in \mathrm{L}_{p}\left(\ell^{\infty}\right)$, where $g(y)=\chi_{B} \operatorname{sgn} y_{1}, \chi_{B}$ is the indicator function of the unit ball $B$ of $\mathbb{R}^{n}$ centered at the origin, and $\left\{x_{j}\right\}_{j \in \mathbb{N}} \subset B$ is a dense sequence in $B$. Since $|R g|$ has arbitrarily large values on some cones, it is easy to check that $\|R f(x, \cdot)\|_{\ell \infty}=\infty$ for all $x \in B$, a contradiction. The same argument 
works if $S=\mathbb{T}$ is the unit circle. We summarize the discussion up to this point in the following proposition.

Proposition 10. The lattice $\mathrm{L}_{\infty}\left(\mathrm{L}_{p}\right)=\mathrm{L}_{\infty}\left(S \times \Omega, \quad \mathrm{L}_{p}\left(\Omega_{1}\right)\right)$ on $\left(S \times \Omega \times \Omega_{1}, \nu \times \mu \times \mu_{1}\right)$ is $\mathrm{A}_{q}$-regular for all $1<p \leq \infty, q>1+\frac{1}{p}$. The lattice $\mathrm{L}_{\infty}\left(\ell^{1}\right)$ on $\mathbb{R}^{n} \times \mathbb{Z}$ or $\mathbb{T} \times \mathbb{Z}$ is not $\mathrm{A}_{p}$-regular for all $1 \leq p<\infty$.

We close this section with a remark on the BMO-regularity of the Lebesgue spaces $\mathrm{L}_{p(\cdot)}$ with variable exponent $p(\cdot)$. These spaces have received considerable attention recently (see, for example, the somewhat dated survey [2], or the comprehensive monograph [21]). For a measurable function $p(\cdot)$ with essinf $p(\cdot)>0$, the space $\mathrm{L}_{p(\cdot)}$ is defined by the Luxemburg quasinorm

$$
\|f\|_{\mathrm{L}_{p(\cdot)}}=\inf \left\{\lambda>0 \mid \int\left(\frac{|f|}{\lambda}\right)^{p(\cdot)} \leq 1\right\} .
$$

Here the following conventions are implied: $|a|^{\infty}=\infty$ for $|a|>1,1^{\infty}=1$, and $0^{\infty}=0$. These spaces are a natural generalization of the standard Lebesgue spaces $\mathrm{L}_{p}$. It is easy to verify the following natural relations: $\mathrm{L}_{p(\cdot)}^{\alpha}=\mathrm{L}_{\frac{p(\cdot)}{\alpha}}$ for $\alpha>0, \mathrm{~L}_{p(\cdot)}^{\prime}=\mathrm{L}_{p^{\prime}(\cdot)}$ if $1 \leq p(\cdot) \leq \infty$ a.e., and $\mathrm{L}_{p(\cdot)} \mathrm{L}_{q(\cdot)}=\mathrm{L}_{s(\cdot)}$ for $\frac{1}{s(\cdot)}=\frac{1}{p(\cdot)}+\frac{1}{q(\cdot)}$. These relations together with Theorem 1 yield the following characterization of BMO-regularity for variable exponent Lebesgue spaces.

Proposition 11. Suppose that $\gamma>1,1 \leq p(\cdot) \leq \infty$ a.e., and $\omega$ is a weight. The following conditions are equivalent.

1) $\mathrm{L}_{p(\cdot)}(\omega)$ is BMO-regular. 2) The maximal operator $M$ is bounded on $\mathrm{L}_{\frac{\gamma p^{\prime}(\cdot)}{p^{\prime}(\cdot)-\alpha}}\left(\omega^{\frac{\alpha}{\gamma}}\right)$ for some (equivalently, for
all) $0<\alpha<1$.

\section{§2. BMO-REGULARITY AND THE BOUNDEDNESS OF THE STANDARD OPERATORS OF HARMONIC ANALYSIS}

In this section we explore the relationship between BMO-regularity and the boundedness of certain operators that often occur in harmonic analysis. Mostly, by that we mean singular integral operators; however, it is convenient to study nonlinear mappings as well. Recall that by our definition $\mathrm{L}_{p}\left(w^{-\frac{1}{p}}\right)$ has the "classical" weighted norm $f \mapsto\left(\int|f|^{p} w\right)^{\frac{1}{p}}$.

Definition 2. A mapping $T$ defined on a set $\Omega_{T}$ of measurable functions on $(S \times \Omega, \nu \times \mu)$ is said to be $\mathrm{A}_{p}$-bounded with constants $(C, m)$ if the $(\nu \times \mu)$-closure of $\Omega_{T}$ contains $\mathrm{L}_{\infty}$ and for any $w \in \mathrm{A}_{p}$ with constant $C$ we have

$$
\|T(f)\|_{\mathrm{L}_{p}\left(w^{-\frac{1}{p}}\right)} \leq m\|f\|_{\mathrm{L}_{p}\left(w^{-\frac{1}{p}}\right)}
$$

for all $f \in \Omega_{T}$. T is weakly $\mathrm{A}_{p^{-}}$bounded if the same is true for all $w \in \mathrm{A}_{1}$ with constant $C$.

In the case of a mapping $T$ acting in the first variable only it suffices to verify $\mathrm{A}_{p^{-}}$ boundedness for a point mass $\mu$. It is well known that (see, e.g. [7]) if $T$ is a singular integral operator bounded on $\mathrm{L}_{2}\left(\mathbb{R}^{n}\right)$, and if its kernel $K(x, y)$ satisfies

$$
|K(x, s)-K(x, t)| \leq C_{K} \frac{|s-t|^{\gamma}}{|x-s|^{n+\gamma}}, \quad x, s, t \in \mathbb{R}^{n}, \quad|x-s|>2|s-t|,
$$


along with the kernel $K(y, x)$ of the adjoint operator for some $\gamma>0,3$ then $T$ is $\mathrm{A}_{p^{-}}$ bounded for all $1<p<\infty$.q We mention that recently the so-called $\mathrm{A}_{2}$ hypothesis was proved in [43, 4 It can be rephrased as follows: all such operators are $\mathrm{A}_{p}$-bounded with constants $\left(C, c_{T, p} C^{\max \{p-1,1\}}\right)$ for all $C>0$, where $c_{T, p}$ depends only on $p$ and the properties of $T$, and this is a sharp estimate for the constants. In other words, for $1<p \leq 2$ (some, not necessarily sharp) constants $m$ of the $\mathrm{A}_{p}$-boundedness of $T$ for a fixed $C$ are of the same magnitude as the (exact for the fixed $C$ ) $\mathrm{A}_{p}$-boundedness constant of the maximal operator $M$.

The $\mathrm{A}_{p}$-boundedness property can often be verified fairly easily. For example, at least in the case of the space $\mathbb{R}^{n}$ with Lebesgue measure, Theorem 2 in 39 states that for all weights $\omega$ and functions $f$ such that $f^{*}(\infty)=0$ (i.e., the measure of all sets $\{|f|>\delta>0\}$ is finite) we have

$$
\int|f|^{p} \omega \leq c_{p, n} \int\left(M_{\lambda_{n}}^{\sharp} f\right)^{p}\left[\frac{M \omega}{\omega}\right]^{p} \omega, \quad 1 \leq p<\infty,
$$

with a constant $c_{p, n}$ for some $\lambda_{n}$. On the right in (4) there is a Fefferman-Stein local sharp maximal function

$$
M_{\lambda}^{\sharp} f(x)=\sup _{Q \ni x} \inf _{c \in \mathbb{R}}\left((f-c) \chi_{Q}\right)^{*}(\lambda|Q|), x \in \mathbb{R}^{n}, \quad 0<\lambda<1 .
$$

The supremum is over all cubes $Q$ containing $x$, the asterisk denotes the nondecreasing rearrangement, and $\chi_{Q}$ is the indicator function of the set $Q$. We obtain the following criterion for the weak $\mathrm{A}_{p}$-boundedness from (4).

Proposition 12. If the mapping $M_{\lambda_{n}}^{\sharp} T$ is $\mathrm{A}_{p}$-bounded with constants $(C, m)$, then $T$ is weakly $\mathrm{A}_{p}$-bounded with constants $\left(C, c_{p, n} m\right)$. In particular, if $M_{\lambda_{n}}^{\sharp} T$ is pointwise dominated by some $\mathrm{A}_{p}$-bounded mapping $S$, i.e., if $\Omega_{T} \subset \Omega_{S}$ and $\left|M_{\lambda_{n}}^{\sharp} T f\right| \leq c|S f|$ for all $f \in \Omega_{T}$, c being a constant independent of $f$, then $T$ is weakly $\mathrm{A}_{p}$-bounded.

The Fefferman-Stein local sharp maximal function is often a convenient tool for pointwise estimates. For any locally integrable $f$, the functions $M^{\sharp} f$ and $M M_{\lambda}^{\sharp} f$ are equivalent a.e. for sufficiently small values of $\lambda$ (see [40]), which means that in Proposition 12 the local sharp maximal function can be replaced by $M^{\sharp}$. Then it is easy to show that the second part of Proposition 12 works for the Calderón-Zygmund operators $T$ due to the well-known pointwise estimate $M^{\sharp} T f \leq c_{r}\left(M|f|^{r}\right)^{\frac{1}{r}}, 1<r<\infty$ (see, e.g., [7. Chapter 4]).

The following result is similar to Proposition 4

Proposition 13. Suppose that $X$ is a Banach lattice on $(S \times \Omega, \nu \times \mu)$ and $1 \leq p<\infty$. Suppose also that $\Omega_{T}$ is dense in $X^{\frac{1}{p}}, X^{\prime}$ is a norming space for $X$, and at least one of the following conditions is satisfied:

(1) $X^{\prime}$ is $\mathrm{A}_{p}$-regular and $T$ is $\mathrm{A}_{p}$-bounded;

(2) $X^{\prime}$ is $\mathrm{A}_{1}$-regular and $T$ is weakly $\mathrm{A}_{p}$-bounded.

Then $T$ is bounded on $X^{\frac{1}{p}}$. If $X$ is BMO-regular, $X$ has the Fatou property, and $T$ is weakly $\mathrm{A}_{p}$-bounded, then $T$ is bounded on $\left(X^{\alpha} \mathrm{L}_{1}^{1-\alpha}\right)^{\frac{1}{p}}$ for all sufficiently small $0<\alpha<1$.

\footnotetext{
${ }^{3}$ Note added in translation. We forgot to mention that this is the usual definition of a CalderónZygmund singular integral operator that we refer to in this paper.

${ }^{4}$ Note added in translation. Now an easier proof is available, presented in a nice self-contained exposition [4].
} 
Observe that if the norm of $X$ is order continuous, then the set $\Omega_{T}$ is always dense in $X^{\frac{1}{p}}$. In order to prove Proposition 13, it suffices to carry out an estimate similar to (11) (or (2) ) for $T$ in place of $M$ and arbitrary functions $f \in \Omega_{T}$ :

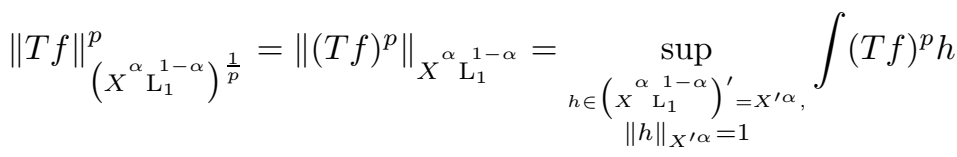

$$
\begin{aligned}
& \leq \sup _{\substack{h \in X^{\prime \alpha},\|h\|_{X^{\prime \alpha}}=1}} \int(T f)^{p} g_{h} \leq \cdots \leq c^{\prime}\|f\|_{X^{\frac{1}{p}}}^{p} .
\end{aligned}
$$

As before, the $g_{h}$ are suitable majorants (the first two cases of Proposition 13 correspond to $\alpha=1$ in (5) ).

Now we turn to the opposite question: to what extent BMO-regularity is characterized by the boundedness of operators? Surely, the operators in question must be nondegenerate in a certain sense.

Definition 3. A mapping $T$ of $\mathrm{L}_{2}$ is said to be BMO-nondegenerate with a constant $C$ if the boundedness of $T$ in the lattice $\mathrm{L}_{2}\left(w^{-\frac{1}{2}}\right)$ implies that $\log w \in$ BMO with the constant $C$. A mapping $T$ is said to be $\mathrm{A}_{p}$-nondegenerate with a constant $C$ if the same implies that $w \in \mathrm{A}_{p}$ with the constant $C$.

Because of the corresponding inclusions, $\mathrm{A}_{p}$-nondegeneracy is stronger than BMOnondegeneracy. The second variable may pose some difficulties when one tries to verify such properties. However, Proposition 19 in $\$ 3$ below shows that if $T$ is a linear operator that is bounded on $\mathrm{L}_{2}$ and is either $\mathrm{A}_{p}$-nondegenerate or BMO-nondegenerate on the measurable space $(S, \nu)$, then the same property is true for the same operator $T$ acting in the first variable for the measurable space $(S \times \Omega, \nu \times \mu)$. The maximal operator is by definition $\mathrm{A}_{2}$-nondegenerate for the measurable space $(S, \nu)$. Many singular integral operators are $\mathrm{A}_{2}$-nondegenerate for the space $(S, \nu)$. For example, any Riesz transformation is $\mathrm{A}_{2}$-nondegenerate for the space $\mathbb{R}^{n}$ with Lebesgue measure (see [7, Chapter 5]).

Theorem 3. Suppose that $X$ is a Banach lattice on $(S \times \Omega, \nu \times \mu)$ with an order continuous norm. If a BMO-nondegenerate operator $T$ is bounded on $X^{\frac{1}{2}}$, then $X^{\prime}$ is $\mathrm{BMO}$ regular with suitable estimates for the constants. If an $\mathrm{A}_{2}$-nondegenerate operator $T$ is bounded on $X^{\frac{1}{2}}$, then $X^{\prime}$ is $\mathrm{A}_{2}$-regular with suitable estimates for the constants, and moreover, $X^{\alpha}$ is $\mathrm{A}_{1}$-regular for all $0<\alpha \leq \alpha_{0}$ with some $\alpha_{0}>\frac{1}{2}$.

Theorem 3 is based on the following theorem.

Theorem 4. Suppose that $Y$ is a Banach lattice on $(S \times \Omega, \nu \times \mu)$ with an order continuous norm. If a linear operator $T$ is bounded on $Y^{\frac{1}{2}}$, then for every $f \in Y^{\prime}, m>1$, and $a>K_{G}\|T\|_{Y^{\frac{1}{2}}}, K_{G}$ being the Grothendieck constant, there exists a majorant $w \geq|f|$, $\|w\|_{Y^{\prime}} \leq \frac{m}{m-1}\|f\|_{Y^{\prime}}$, such that $\|T\|_{\mathrm{L}_{2}\left(w^{-\frac{1}{2}}\right)} \leq a \sqrt{m}$.

The proof of Theorem 4 is given below in 96 . This is, essentially, a known result (see [46] and [1]).

Naturally, Theorem 4 means that if, under the assumptions of Theorem 3 , the operator $T$ is bounded on $X^{\frac{1}{2}}$, then $X^{\prime}$ is BMO-regular by the BMO-nondegeneracy of $T$. If $T$ is $\mathrm{A}_{2}$-nondegenerate, then an application of Theorem 4 shows that $X^{\prime}$ is $\mathrm{A}_{2}$-regular, and by Proposition 4 the lattice $X^{\delta}$ is $\mathrm{A}_{1}$-regular for all $0<\delta<\alpha_{0}$ and some $\alpha_{0}>\frac{1}{2}$, as claimed. 
Note that if $X$ is a Banach lattice, then $X^{\alpha} \mathrm{L}_{1}^{1-\alpha}$ has order continous norm for $0<$ $\alpha<1$ because $\mathrm{L}_{1}^{1-\alpha}=\mathrm{L}_{\frac{1}{1-\alpha}}$ has order continuous norm. Combining Proposition 13 and Theorem 3, we get the following result.

Theorem 5. Suppose that $X$ is a Banach lattice with the Fatou property on $(S \times \Omega, \nu \times \mu)$. The following conditions are equivalent.

1) $X$ is BMO-regular.

2) Some weakly $\mathrm{A}_{2}$-bounded and $\mathrm{BMO}$-nondegenerate linear operator $T$ is bounded on $\left(X^{\alpha} \mathrm{L}_{1}^{1-\alpha}\right)^{\frac{1}{2}}$ for some $0<\alpha<1$.

3) All weakly $\mathrm{A}_{2}$-bounded mappings $T$ are bounded on $\left(X^{\alpha} \mathrm{L}_{1}^{1-\alpha}\right)^{\frac{1}{2}}$ for all sufficiently small values of $0<\alpha<1$.

We emphasize again that $T$ in condition 3 ) can be any of the Calderón-Zygmund type singular integral operators, and in condition 2) in the case of lattices on $\mathbb{R}^{n} \times \Omega$ any of the Riesz transformations can be substituted for $T$.

\section{§3. Auxiliary statements}

In this section we state the main results that we need for the proofs, along with some auxiliary propositions. The proof of Theorem 2 in $\$ 4$ below and the proof of a similar result in $\$$ are based on application of a fixed point theorem.

Theorem (Ky Fan-Kakutani [23). Suppose that $K$ is a compact set in a locally convex linear topological space. Let $\Phi$ be a mapping from $K$ to the set of nonempty compact convex subsets of $K$. If the graph $\{(x, y) \in K \times K \mid y \in \Phi(x)\}$ of $\Phi$ is closed in $K \times K$, then $\Phi$ has a fixed point, i.e., $x \in \Phi(x)$ for some $x \in K$.

The proof of Theorem 4 given below in $\$$ makes use of the following result, which is usually referred to as the Grothendieck theorem for lattices (see, e.g., 9]).

Theorem (see [41]). Suppose that $X$ and $Y$ are Banach lattices and $T: X \rightarrow Y$ is a bounded linear operator. Then

$$
\left\|\left(\sum_{j=1}^{n}\left|T x_{j}\right|^{2}\right)^{\frac{1}{2}}\right\|_{Y} \leq K_{G}\|T\|_{X \rightarrow Y}\left\|\left(\sum_{j=1}^{n}\left|x_{j}\right|^{2}\right)^{\frac{1}{2}}\right\|_{X}
$$

for any finite set $\left\{x_{j}\right\}_{j=1}^{n} \subset X$, where $K_{G}$ is the universal Grothendieck constant.

The following theorem plays an important part in the sequel. It means that in a lattice with the Fatou property, the sets that are convex, bounded, and closed in measure (i.e., with respect to convergence in measure on all sets of finite measure) share certain properties with compact sets.

Theorem 6 (see [18, Theorem 3, Chapter X, $\S 6]$ ). Let $X$ be a Banach lattice with the Fatou property on $(\Sigma, \mu)$ and $\left\{V_{\xi}\right\}_{\xi \in \Xi}$ a centered family of sets in $X$ that are convex, bounded, and $\mu$-closed. Then $\bigcap_{\xi \in \Xi} V_{\xi}$ is not empty.

The following simple proposition makes it possible to replace a function with a slightly larger one that is positive a.e. with only a slight increase in norm.

Proposition 14. Suppose that $X$ is a Banach lattice on $(\Sigma, \mu)$. Then for every $f \in X$ such that $f \neq 0$ identically and every $\varepsilon>0$ there exists $g \in X$ such that $g>|f|$ a.e. and $\|g\|_{X} \leq(1+\varepsilon)\|f\|_{X}$. 
For the proof it suffices to take any representation $\Sigma=\bigcup_{j} A_{j}$, where $A_{j}$ is a nondecreasing sequence of sets of finite measure such that $\chi_{A_{j}} \in X$ (see, e.g., [18, Chapter IV, $\S 3$, Corollary 2]) and put

$$
g=|f|+\varepsilon \sum_{j \geq 1} 2^{-j} \frac{\chi_{A_{j}}}{\left\|\chi_{A_{j}}\right\|_{X}} .
$$

The following proposition is well known (see, e.g., [12, 1.2]).

Proposition 15. Suppose $X$ is a Banach lattice with the Fatou property on $(\Sigma, \mu)$ and $F_{n} \subset X$ is a decreasing sequence of sets in $X$ that are bounded, convex, and $\mu$-closed. Then for any sequence $f_{n} \in F_{n}$ there exists a sequence of finite convex combinations

$$
g_{n}=\sum_{k \geq n} \alpha_{k}^{(n)} f_{k}, \quad \alpha_{k}^{(n)} \geq 0, \quad \sum_{k \geq n} \alpha_{k}^{(n)}=1,
$$

such that $g_{n}$ converge a.e. to some $f \in F=\bigcap_{n} F_{n}$.

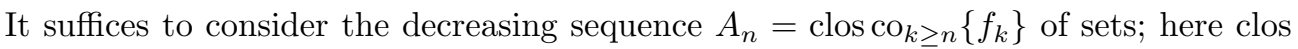
denotes closure in measure and co denotes the convex hull. By the assumptions of the proposition, $A_{n} \subset F_{n}$ for all $n$, so Proposition [6 applied to this family shows that $\bigcap_{n} A_{n}$ is not empty. Take any $f \in \bigcap_{n} A_{n}$. Then $f \in F$ and for every $n$ there exists a sequence $g_{k}^{n} \in \mathrm{co}_{k \geq n}\left\{f_{k}\right\}$ such that $g_{k}^{n}$ converges to $f$ in measure on all sets of finite measure as $k \rightarrow \infty$. Since convergence in measure is metrizable, we can easily find a sequence of indices $k_{n}$ such that the sequence $g_{k_{n}}^{n}$ converges to $f$ in measure on all sets of finite measure. Therefore, some subsequence of this sequence converges to $f$ almost everywhere, which concludes the proof of Proposition 15.

Suppose that $1 \leq p<\infty$ and $C \geq\|M\|_{\mathrm{L}_{p}}$. We introduce the following sets of nonnegative a.e. measurable functions $w$ on $(S \times \Omega, \nu \times \mu)$ :

$$
\begin{aligned}
& B \mathrm{~A}_{p}(C)=\left\{w \mid \underset{\omega \in \Omega}{\operatorname{ess} \sup }\|M\|_{\mathrm{L}_{p}\left(w^{\left.-\frac{1}{p}(\cdot, \omega)\right)}\right.} \leq C\right\}, \quad p>1 ; \\
& B \mathrm{~A}_{1}(C)=\left\{w \mid \operatorname{ess} \operatorname{esp} \frac{M w}{w} \leq C\right\} .
\end{aligned}
$$

These are the sets of Muckenhoupt weights with given bounds on the constants ("the Ball of $\mathrm{A}_{p}$ "). The following proposition (cf. [22, Lemma 4.2]) shows that their geometric structure is simple and convenient.

Proposition 16. Suppose that $1 \leq p<\infty$ and $C \geq 0$. The set $B \mathrm{~A}_{p}(C)$ is a nonempty convex cone; also, it is logarithmically convex and closed in measure. The natural relation $\bigcap_{c>C} B \mathrm{~A}_{p}(c)=B \mathrm{~A}_{p}(C)$ holds true.

The additivity and homogeneity of the sets $B \mathrm{~A}_{p}(C)$, and consequently, their convexity, are quite obvious. Nonemptiness is also obvious because $0 \in B \mathrm{~A}_{p}(C)$. We prove that $B \mathrm{~A}_{p}(C)$ is closed in measure. Suppose that a sequence $w_{j} \in B \mathrm{~A}_{p}(C)$ converges in measure on all sets of finite measure to some $w$. By passing to a subsequence, we can assume that the $w_{j}$ converge to $w$ almost everywhere. If $p=1$, the Fatou theorem yields immediately

$$
\frac{1}{\nu(B)} \int_{B} w \leq \frac{1}{\nu(B)} \liminf _{n} \int_{B} w_{n}
$$

for any ball $B$, whence

$$
\frac{M w}{w} \leq \frac{\liminf _{n} M w_{n}}{w} \leq C \frac{\liminf _{n} w_{n}}{w}=C
$$


a.e., and $w \in A B_{1}(C)$. Now we turn to the case where $p>1$. Note that $W=\sup _{j} w_{j}$ is finite almost everywhere. Since $\mathrm{L}_{p}\left(W^{-\frac{1}{p}}\right)$ is dense in measure and in norm in the space 5 $\mathrm{L}_{p}\left(w^{-\frac{1}{p}}\right)$, it is suffices to verify that

$$
\|M f\|_{\mathrm{L}_{p}\left(w^{-\frac{1}{p}}\right)} \leq C\|f\|_{\mathrm{L}_{p}\left(w^{-\frac{1}{p}}\right)}
$$

for any $f \in \mathrm{L}_{p}\left(W^{-\frac{1}{p}}\right)$. It is clear that $f \in \mathrm{L}_{p}\left(w_{j}^{-\frac{1}{p}}\right)$ for all $j$. Thus,

$$
\int_{S}(M f(t, \omega))^{p} w_{j}(t, \omega) d \nu(t) \leq C^{p} \int_{S}|f(t, \omega)|^{p} w_{j}(t, \omega) d \nu(t)
$$

for a.e. $\omega \in \Omega$ and all $j$. Passing to the limit in (6) and making use of the Fatou theorem and the Lebesgue dominated convergence theorem, we arrive at the inequality

$$
\begin{aligned}
\int_{S}(M f(t, \omega))^{p} w d \nu(t) & \leq \liminf _{j \rightarrow \infty} \int_{S}(M f(t, \omega))^{p} w_{j}(t, \omega) d \nu(t) \\
& \leq \lim _{j \rightarrow \infty} C^{p} \int_{S}\left[\frac{w_{j}(t, \omega)}{W(t, \omega)}\right]|f(t, \omega)|^{p} W(t, \omega) d \nu(t) \\
& =C^{p} \int_{S}|f(t, \omega)|^{p} w(t, \omega) d \nu(t)
\end{aligned}
$$

for a.e. $\omega \in \Omega$ and $w \in B \mathrm{~A}_{p}(C)$, which shows that the set $B \mathrm{~A}_{p}(C)$ is indeed closed in measure.

We verify the logarithmic convexity of $B \mathrm{~A}_{p}(C)$. Suppose that $0<\theta<1$ and $w_{0}, w_{1} \in$ $B \mathrm{~A}_{p}(C)$; we need to prove that

$$
w=w_{\theta}=w_{0}^{\theta} w_{1}^{1-\theta} \in B \mathrm{~A}_{p}(C) .
$$

Observe that it suffices to establish (8) for $\theta=\frac{1}{2}$, because then we can reiterate this inequality to get (8) for all binary fractions $\theta$ and then obtain (8) for arbitrary $0<\theta<1$ by taking a sequence $\theta_{n} \rightarrow \theta$ and using the measure closedness of $B \mathrm{~A}_{p}(C)$. Now suppose that $\theta=\frac{1}{2}$ and $f \in \mathrm{L}_{p}\left(w^{-\frac{1}{p}}\right)$. The Cauchy-Schwarz inequality easily yields

$$
\begin{aligned}
M f(x, t)= & \sup _{B \ni x} \frac{1}{\nu(B)} \int_{B}\left[|f(y, t)|\left(\frac{w_{1}(y, t)}{w_{0}(y, t)}\right)^{\frac{1}{2 p}}\right]^{\frac{1}{2}}\left[|f(y, t)|\left(\frac{w_{0}(y, t)}{w_{1}(y, t)}\right)^{\frac{1}{2 p}}\right]^{\frac{1}{2}} d \nu(y) \\
\leq & {\left[\sup _{B \ni x} \frac{1}{\nu(B)} \int_{B}|f(y, t)|\left(\frac{w_{1}(y, t)}{w_{0}(y, t)}\right)^{\frac{1}{2 p}} d \nu(y)\right]^{\frac{1}{2}} } \\
& \times\left[\sup _{B \ni x} \frac{1}{\nu(B)} \int_{B}|f(y, t)|\left(\frac{w_{0}(y, t)}{w_{1}(y, t)}\right)^{\frac{1}{2 p}} d \nu(y)\right]^{\frac{1}{2}} \\
= & {\left[M\left[f\left(\frac{w_{1}}{w_{0}}\right)^{\frac{1}{2 p}}\right](x, t)\right]^{\frac{1}{2}}\left[M\left[f\left(\frac{w_{0}}{w_{1}}\right)^{\frac{1}{2 p}}\right](x, t)\right]^{\frac{1}{2}} }
\end{aligned}
$$

\footnotetext{
${ }^{5}$ Note added in translation. Generally speaking, under our corrected definition of the weighted spaces (see the footnote in 41) $\mathrm{L}_{p}\left(W^{-\frac{1}{p}}\right)$ is not contained in $\mathrm{L}_{p}\left(w^{-\frac{1}{p}}\right)$ even though $W \geq w$ almost everywhere. Thus, we should replace this set with $\mathrm{L}_{p}\left(W^{-\frac{1}{p}}\right) \cap \mathrm{L}_{p}\left(w^{-\frac{1}{p}}\right)$ and also replace "norm" with "quasinorm" in the proof to rectify this minor inaccuracy.
} 
for $x \in S$ and $t \in \Omega$. In case $p>1$, another application of the Cauchy-Schwarz inequality leads to

$$
\begin{aligned}
\|M f\|_{\mathrm{L}_{p}\left(w^{-\frac{1}{p}}\right)}^{p} & =\int(M f)^{p} w \\
& \leq \int\left[\left(M\left[f\left(\frac{w_{1}}{w_{0}}\right)^{\frac{1}{2 p}}\right]\right)^{p} w_{0}\right]^{\frac{1}{2}}\left[\left(M\left[f\left(\frac{w_{0}}{w_{1}}\right)^{\frac{1}{2 p}}\right]\right)^{p} w_{1}\right]^{\frac{1}{2}} \\
& \leq\left[\int\left(M\left[f\left(\frac{w_{1}}{w_{0}}\right)^{\frac{1}{2 p}}\right]\right)^{p} w_{0}\right]^{\frac{1}{2}}\left[\int\left(M\left[f\left(\frac{w_{0}}{w_{1}}\right)^{\frac{1}{2 p}}\right]\right)^{p} w_{1}\right]^{\frac{1}{2}} \\
& \leq\left(C^{p} \int\left[|f|^{p}\left(\frac{w_{1}}{w_{0}}\right)\right]^{\frac{1}{2}} w_{0}\right)^{\frac{1}{2}}\left(C^{p} \int\left[|f|^{p}\left(\frac{w_{0}}{w_{1}}\right)\right]^{\frac{1}{2}} w_{1}\right)^{\frac{1}{2}} \\
& =C^{p} \int|f|^{p} w_{0}^{\frac{1}{2}} w_{1}^{\frac{1}{2}}=C^{p}\|f\|_{\mathrm{L}_{p}\left(w^{-\frac{1}{p}}\right)}^{p}
\end{aligned}
$$

For $p=1$ estimate (9) with $f=w$ shows that

$$
\frac{M w}{w} \leq\left(\frac{M w_{0}}{w_{0}}\right)^{\frac{1}{2}}\left(\frac{M w_{1}}{w_{1}}\right)^{\frac{1}{2}} \leq C
$$

almost everywhere. Probably, such estimates can be obtained for any $0<\theta<1$ directly, by using the Hölder inequality 6 Finally, the relation $\bigcap_{c>C} B \mathrm{~A}_{p}(c)=B \mathrm{~A}_{p}(C)$ is trivial, which concludes the proof of Proposition 16.

\footnotetext{
${ }^{6}$ Note added in translation. Indeed, this is quite easy, but requires a somewhat longer and less clear computation. Suppose that $u$ is positive on the support of $w$ and $f$ is 0 a.e. outside of the support of $w$. The Hölder inequality yields

$$
\begin{aligned}
& \frac{1}{\nu(B)} \int_{B}|f(x, t)| d \nu(t)=\frac{1}{\nu(B)} \int_{B}|f(x, t)|^{\theta} u(x, t)|f(x, t)|^{1-\theta}[u(x, t)]^{-1} d \nu(t) \\
& \quad \leq\left(\frac{1}{\nu(B)} \int_{B}|f(x, t)|[u(x, t)]^{\frac{1}{\theta}} d \nu(t)\right)^{\theta}\left(\frac{1}{\nu(B)} \int_{B}|f(x, t)|[u(x, t)]^{-\frac{1}{1-\theta}} d \nu(t)\right)^{1-\theta}
\end{aligned}
$$
}

for any ball $B$, whence

$$
M f \leq\left[M\left(f \cdot u^{\frac{1}{\theta}}\right)\right]^{\theta}\left[M\left(f \cdot u^{-\frac{1}{1-\theta}}\right)\right]^{1-\theta}
$$

almost everywhere. Another use of the Hölder inequality produces the estimate

$$
\begin{aligned}
\left.\|M f\|_{\mathrm{L}_{p}(w}^{p}-\frac{1}{p}\right) & \leq \int\left[M\left(f \cdot u^{\frac{1}{\theta}}\right)\right]^{p \theta}\left[M\left(f \cdot u^{-\frac{1}{1-\theta}}\right)\right]^{p(1-\theta)} w \\
& \leq\left(\int\left[M\left(f \cdot u^{\frac{1}{\theta}}\right)\right]^{p} w\right)^{\theta}\left(\int\left[M\left(f \cdot u^{-\frac{1}{1-\theta}}\right)\right]^{p} w\right)^{1-\theta} \\
& \leq C^{p}\left(\int|f|^{p} u^{\frac{p}{\theta}} w\right)^{\theta}\left(\int|f|^{p} u^{-\frac{p}{1-\theta}} w\right)^{1-\theta} .
\end{aligned}
$$

Clearly, it suffices to find some $u$ that satisfies both $u^{\frac{p}{\theta}} w=w_{0}$ and $u^{-\frac{p}{1-\theta}} w=w_{1}$ simultaneously. These two relations are equivalent and can be rewritten as $u=\left(\frac{w_{0}}{w_{1}}\right)^{\frac{\theta(1-\theta)}{p}}$. Substituting this in the above estimates, we arrive at the logarithmic convexity of $B \mathrm{~A}_{p}(C)$ for $p>1$. For $p=1$ and $f=w$, estimate (10) yields

$$
M w \leq\left(M w_{0}\right)^{\theta}\left(M w_{1}\right)^{1-\theta} \leq C w_{0}^{\theta} w_{1}^{1-\theta}
$$

almost everywhere, which corresponds to the logarithmic convexity of $B \mathrm{~A}_{p}(C)$ for $p=1$. 
We introduce the set of $\mathrm{A}_{p}$-majorants

$$
M_{C, m}^{X, p}(f)=\left\{g\left|\|g\|_{X} \leq m, g \geq\right| f \mid\right\} \cap B \mathrm{~A}_{p}(C)
$$

for any Banach lattice $X$ satisfying the Fatou property and a function $f \in X$ nonnegative a.e. The nonemptiness of this set characterizes the existence of an $\mathrm{A}_{p}$-majorant for a given function $f$ with constants $(C, m)$ provided that $\|f\|_{X}=1$. Let us explore certain properties of the set (111). The Fatou property and Proposition 16 imply that $M_{C, m}^{X, p}(f) \subset X$ is closed in measure, convex, and logarithmically convex (see also (13) below). It is easily seen that $M_{C, m}^{X, p}(f)$ is monotone in $f$, i.e.,

$$
M_{C, m}^{X, p}(f) \supset M_{C, m}^{X, p}\left(f_{1}\right)
$$

for any $f_{1} \geq|f|$. It is well known that the unit ball of any Banach lattice $X$ is logarithmically convex. Moreover, for such a lattice there is a version of the Hölder inequality:

$$
\|a b\|_{X} \leq\left\||a|^{r}\right\|_{X}^{\frac{1}{r}}\left\||b|^{r^{\prime}}\right\|_{X}^{\frac{1}{r^{\prime}}}, \quad 1<r<\infty .
$$

Using (13), it is easy to show that

$$
\prod_{j=1}^{n}\left[M_{C, m}^{X, p}\left(f_{j}\right)\right]^{\theta_{j}} \subset M_{C, m}^{X, p}\left(\prod_{j=1}^{n}\left|f_{j}\right|^{\theta_{j}}\right)
$$

for all $\theta_{j}>0, \sum_{j=1}^{n} \theta_{j}=1$, and $f_{j} \in X$. On the left-hand side of (14) multiplication and exponentiation are understood pointwise. Setting $f_{j}=f$ in (14) yields the logarithmic convexity of the set $M_{C, m}^{X, p}(f)$.

Proposition 17. Suppose $X$ is a lattice with the Fatou property on $(S \times \Omega, \nu \times \mu)$ and $f_{j} \in X, f_{j} \geq 0$ a.e., constitute a monotone nondecreasing sequence converging a.e. to some $f \in X$. Then the intersection $\bigcap_{j} M_{C, m}^{X, p}\left(f_{j}\right)$ coincides with $M_{C, m}^{X, p}(f)$. If, additionally, the sets $M_{C, m}^{X}\left(f_{j}\right)$ are nonempty for all $j$, then the set $M_{C, m}^{X}(f)$ is also nonempty.

The inclusion $\bigcap_{j} M_{C, m}^{X, p}\left(f_{j}\right) \supset M_{C, m}^{X, p}(f)$ follows from (12). For any $g$ in $\bigcap_{j} M_{C, m}^{X, p}\left(f_{j}\right)$ we have $\|g\|_{X} \leq m,|g| \geq\left|f_{j}\right|$. Passing to the limit in the last estimate yields $g \in$ $M_{C, m}^{X, p}(f)$, so that the reverse inclusion is also true. Finally, the sets $M_{C, m}^{X, p}\left(f_{j}\right)$ are closed in measure, convex, and bounded in $X$, and they form a nonincreasing sequence. Therefore, if they are nonempty, Theorem $\left[6\right.$ shows that their intersection $M_{C, m}^{X, p}\left(f_{j}\right)$ is also nonempty. This concludes the proof of Proposition 17.

The following proposition shows that for lattices with the Fatou property it suffices to verify $\mathrm{A}_{p}$-regularity on functions belonging to some dense set; moreover, the constants can be relaxed slightly.

Proposition 18. Suppose that $X$ is a Banach lattice with the Fatou property on $(S \times \Omega, \nu \times \mu)$. Suppose also that a set $F \subset S_{+}=\left\{f \in X_{+} \mid\|f\|_{X} \leq 1\right\}$ has the following property: for all $\alpha>0$ and $f \in F$, there exists some $g \in X$ such that $g \geq|f|,\|g\|_{X} \leq m+\alpha$, and $g \in \mathrm{A}_{p}$ with constant $C+\alpha$. Then all functions in the set $\left\{f \mid\|f\|_{X}=1\right\} \cap \bar{F}$, where $\bar{F}$ denotes the closure of $F$ in measure, have $\mathrm{A}_{p}$-majorants in $X$ with constants $(C, m)$.

Suppose that a function $0 \leq f \in X,\|f\|_{X}=1$, belongs to the closure of $F$ in measure. Then there is some sequence $f_{n} \in F$ converging to $f$ almost everywhere. By the assumptions of the proposition, there are some $g_{n} \in M_{C+\frac{1}{n}, m+\frac{1}{n}}^{X, p}\left(f_{n}\right) \subset M_{C+\frac{1}{n}, m+\frac{1}{n}}^{X, p}(0)$, so by Proposition 15 there exists $g \in \cap_{n} M_{C+\frac{1}{n}, m+\frac{1}{n}}^{X, p}(0)=M_{C, m}^{X, p}(0)$ (equality follows 
from Proposition (16) and a sequence of convex combinations $G_{n}=\sum_{j \geq n} \alpha_{j}^{(n)} g_{n}$ that converges to $g$ almost everywhere. Since

$$
G_{n} \geq F_{n}=\sum_{j \geq n} \alpha_{j}^{(n)} f_{n} \rightarrow f
$$

almost everywhere, passing to the limit yields $g \geq f$, whence $g \in M_{C, m}^{X, p}(f)$. The proof of Proposition 18 is complete.

Proposition 19. Suppose that a linear operator $T$ is bounded on $\mathrm{L}_{2}$ on a measurable space $(S, \nu)$. If $T$ is $\mathrm{A}_{p}$-nondegenerate, then the same operator $T$ acting in the first variable is also $\mathrm{A}_{p}$-nondegenerate for a measurable space $(S \times \Omega, \nu \times \mu)$. The same is true for BMO-nondegeneracy.

Suppose that for some function $w$ positive a.e. on $(S \times \Omega, \nu \times \mu)$ we have

$$
\|T\|_{\mathrm{L}_{2}\left(w^{-\frac{1}{2}}\right)} \leq C
$$

it suffices to prove that

$$
\|T\|_{\mathrm{L}_{2}\left(w^{-\frac{1}{2}}(\cdot, \omega)\right)} \leq C
$$

for a.e. $\omega \in \Omega$. First, we establish this under the assumption that $w$ is essentially bounded. Then we have the continuous inclusion $\mathrm{L}_{2}(S, \nu) \subset \mathrm{L}_{2}\left(w^{-\frac{1}{2}}(\cdot, \omega)\right)$ for almost every $\omega \in \Omega$, and also $\mathrm{L}_{2}(S, \nu)$ is separable and dense in $\mathrm{L}_{2}\left(w^{-\frac{1}{2}}(\cdot, \omega)\right)$. Denote by $\left\{f_{j}\right\}$ some dense sequence in $\mathrm{L}_{2}(S, \nu)$; then this sequence is also dense in $\mathrm{L}_{2}\left(w^{-\frac{1}{2}}(\cdot, \omega)\right)$. If condition (16) is not satisfied on some set $E \subset \Omega$ of positive measure $\mu$, then for every $\omega \in E$ there exists an index $j_{\omega}$ such that

$$
\int\left|T f_{\omega_{j}}(s)\right|^{2} w(s, \omega) d \nu(s)>C^{2} \int\left|f_{\omega_{j}}\right|^{2} w(s, \omega) d \nu(s) .
$$

Since the sets $E_{j}=\left\{\omega \mid j_{\omega}=j\right\}$ are measurable and $E=\bigcup_{j} E_{j}$, there exists an index $j$ such that $\mu\left(E_{j}\right)>0$. However, this means that for $f=\chi_{E_{j}} f_{j}$ we have

$$
\int|T f|^{2} w>C^{2} \int|f|^{2} w
$$

which contradicts (15)). Therefore, for bounded weights $w$ the transition from (15) to (16) is justified.

Now observe that, under the assumptions of Proposition [19, the operator $T$ is uniformly bounded on the lattices $\mathrm{L}_{2}\left(w_{n}^{-\frac{1}{2}}\right)$ with the weights $w_{n}=w \wedge n$. This is a consequence of the following simple norm estimate for the conjugate operator $T^{*}$ (conjugation is understood in the sense of the weightless $\mathrm{L}_{2}$ space duality) in the lattice $\left[\mathrm{L}_{2}\left(w_{n}^{-\frac{1}{2}}\right)\right]^{*}=\left[\mathrm{L}_{2}\left(w_{n}^{-\frac{1}{2}}\right)\right]^{\prime}=\mathrm{L}_{2}\left(w_{n}^{\frac{1}{2}}\right):$

$$
\begin{aligned}
\left\|T^{*} f\right\|_{\mathrm{L}_{2}\left(w_{n}^{\frac{1}{2}}\right)}^{2} & =\int\left|T^{*} f\right|^{2}\left(w^{-1} \vee \frac{1}{n}\right) \leq \int\left|T^{*} f\right|^{2} w^{-1}+\frac{1}{n} \int\left|T^{*} f\right|^{2} \\
& \leq c^{2}\left(\int|f|^{2}\left[w^{-1}+\frac{1}{n}\right]\right) \leq 2 c^{2} \int|f|^{2}\left(w^{-1} \vee \frac{1}{n}\right) \\
& =2 c^{2}\left\|T^{*} f\right\|_{\mathrm{L}_{2}\left(w_{n}^{\frac{1}{2}}\right)}^{2},
\end{aligned}
$$

where $f \in \mathrm{L}_{2}\left(w_{n}^{\frac{1}{2}}\right)$ and

$$
c=\left\|T^{*}\right\|_{\mathrm{L}_{2}} \vee\left\|T^{*}\right\|_{\mathrm{L}_{2}\left(w^{\frac{1}{2}}\right)}=\|T\|_{\mathrm{L}_{2}} \vee\|T\|_{\mathrm{L}_{2}\left(w^{-\frac{1}{2}}\right)} .
$$


Thus, we can use the transition from (15) to (16) already established for the weight $w_{n}$ to obtain

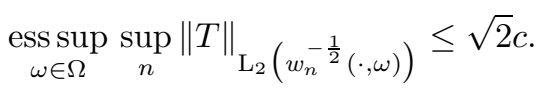

Finally, it is possible to pass to the limit in $n$ in the estimate

$$
\int|T f(x)|^{2} w_{n}(x, \omega) d \nu(x) \leq 2 c^{2} \int|T f(x)|^{2} w_{n}(x, \omega) d \nu(x), \quad f \in \mathrm{L}_{2}\left(w^{-\frac{1}{2}}\right),
$$

using the monotone continuity of the Lebesgue integral. This yields the required estimate (16) for $C=\sqrt{2} c$, which concludes the proof of Proposition 19 ,

\section{$\S 4$. Proof of Theorem 2}

In this section we present the proof of Theorem 2, The proof is based on a similar result [26] for couples of lattices on the unit circle, which we also establish for the general case in $\$ 5$ below along with another proof of Theorem 2. The two proofs of Theorem 2 are essentially identical in the case of a single lattice. We give the proof for the case of a single lattice separately because it looks more natural, a bit simpler (at least conceptually if not technically), and it makes the exposition of the BMO-regularity theory for a single lattice given in the preceding sections of this paper independent of similar results for couples that we give below in $\$ 5$.

Suppose that, under the assumptions of Theorem 2, the lattice $X \mathrm{~L}_{q}$ is $\mathrm{A}_{p}$-regular with constants $(C, m)$. We may assume that $\mu(\Omega)=1$. Indeed, because we can replace the measure $\mu$ by an equivalent one that has this property, because a weight that is constant in the first variable for a.e. value of the second variable has no effect on $A_{p}$-regularity. Since the set of functions $f$ in $X$ having support in $B=B_{0} \times \Omega$ for some ball $B_{0}$ of the space $(S, \nu)$ is dense in measure in the lattice $X$, by Proposition 18 it suffices to establish the existence of $\mathrm{A}_{p+1}$-majorants for all such functions $f$ with some sufficiently large fixed constants $\left(C_{1}, m_{1}\right)$ to be determined later. The lattice $\mathrm{L}_{q}$ is $\mathrm{A}_{1}$-regular with some constants $\left(C_{q}, m_{q}\right)$. We may assume that $m_{1}>m m_{q}$. Put

$$
D=\left\{\log h \mid\|h\|_{\mathrm{L}_{q}} \leq 1, h \in B \mathrm{~A}_{1}\left(C_{q}\right), h \geq \chi_{B} \delta\right\}
$$

for all sufficiently small $\delta>0$. Recall that $B \mathrm{~A}_{1}\left(C_{q}\right)=\left\{w \mid M w \leq C_{q} w\right\}$ (see the definition before Proposition (16). It is well known that $h=\left(M_{\chi_{B}}\right)^{\beta}$ belongs to $\mathrm{A}_{1}$ if $0<\beta<1$ (see [7, Chapter 5, 5.2]) with some constant $C^{\prime}$ independent of a set $B$. Surely, $h \in \mathrm{L}_{q}$ for $\beta>\frac{1}{q}$. We fix some $\beta$ satisfying $\frac{1}{q}<\beta<1$. Assuming that $C_{q} \geq C^{\prime}$ and $0<\delta<\|h\|_{\mathrm{L}_{q}}^{-1}$, we see that $\log \left(\frac{h}{\|h\|_{\mathrm{L}_{q}}}\right) \in D$, so $D$ is nonempty. The set $D$ is also convex and closed in measure, due to the Hölder inequality and Proposition 16.

Without loss of generality we may assume that $\|f\|_{X}=1$. The $\mathrm{A}_{p}$-regularity of $X \mathrm{~L}_{q}$ means that for any $\log u \in D$ the set of the corresponding $\mathrm{A}_{p}$-majorants $M_{C, m}^{X \mathrm{~L}_{q}, p}(u f)$ is nonempty (for the definition of this set, see (11) in \$3). We recall the definition of the norm in the product of a couple of quasinormed lattices $Y$ and $Z$ :

$$
\|x\|_{Y Z}=\inf _{x=a b}\|a\|_{Y}\|b\|_{Z}=\inf _{\|b\|_{Z}=1}\left\|x b^{-1}\right\|_{Y} .
$$

For any $\gamma>0$ and a couple of lattices $Z \ni \chi_{B}$ and $Y$, we can write

$$
\begin{aligned}
\|x\|_{Y Z} & =\inf _{\|b\|_{Z}=1}\left\|x b^{-1}\right\|_{Y}\left\|b \vee \gamma \chi_{B}\right\|_{Z} \frac{\|b\|_{Z}}{\left\|b \vee \gamma \chi_{B}\right\|_{Z}} \\
& \geq\left(1+\left\|\gamma \chi_{B}\right\|_{Z}\right)^{-1} \inf _{\|b\|_{Z}=1}\left\|x\left(b \vee \gamma \chi_{B}\right)^{-1}\right\|_{Y}\left\|b \vee \gamma \chi_{B}\right\|_{Z} \\
& \geq\left(1+\left\|\gamma \chi_{B}\right\|_{Z}\right)^{-1} \inf _{\substack{\|b\|_{Z} \leq 1, b \geq \gamma \chi_{B}}}\left\|x b^{-1}\right\|_{Y} .
\end{aligned}
$$


The first inequality in this estimate can be explained as follows: for any $\|b\|_{Z}=1$ we have

$$
\frac{\left\|b \vee \gamma \chi_{B}\right\|_{Z}}{\|b\|_{Z}} \leq \frac{\|b\|_{Z}+\left\|\gamma \chi_{B}\right\|_{Z}}{\|b\|_{Z}}=1+\left\|\gamma \chi_{B} 1\right\|_{Z}
$$

We may assume $\delta$ to be so small that $m m_{q}\left(1+\left\|m_{q} \delta \chi_{B}\right\|_{\mathrm{L}_{q}}\right) \leq m_{1}$. Using estimate (17) and the $\mathrm{A}_{1}$-regularity of $\mathrm{L}_{q}$ mentioned above, we see that if $\log u \in D$ and $g \in$ $M_{C, m}^{X \mathrm{~L}_{q}, p}(f u)$, then

$$
\begin{aligned}
m \geq\|g\|_{X \mathrm{~L}_{q}} & \geq\left(1+\left\|m_{q} \delta \chi_{B}\right\|_{\mathrm{L}_{q}}\right)^{-1} \inf _{\substack{\|b\|_{L_{q}} \leq 1, b \geq m_{q} \delta \chi_{B}}}\left\|g b^{-1}\right\|_{X} \\
& \geq\left(1+\left\|m_{q} \delta \chi_{B}\right\|_{\mathrm{L}_{q}}\right)^{-1} m_{q}^{-1} \inf _{\log a \in D}\left\|g a^{-1}\right\|_{X} ;
\end{aligned}
$$

the last inequality is obtained by replacing $b$ with its respective $\mathrm{A}_{1}$-majorant $a$ with constants $\left(C_{q}, m_{q}\right)$. Estimate (18) means that for any $\log u \in D$ the set

$$
\Phi(\log u)=\{\log a \in D \mid M(u, a) \neq \varnothing\},
$$

where

$$
M(u, a)=\left\{g \in M_{C, m}^{X \mathrm{~L}_{q}, p}(f u) \mid\left\|g a^{-1}\right\|_{X} \leq m_{1}\right\},
$$

is nonempty. It is easily seen that all sets $M(u, a)$ are convex, bounded in $X \mathrm{~L}_{q}$, and closed in measure. From (14) and (13) we obtain

$$
\prod_{j=1}^{n}\left[M\left(u_{j}, a_{j}\right)\right]^{\theta_{j}} \subset M\left(\prod_{j=1}^{n}\left|u_{j}\right|^{\theta_{j}}, \prod_{j=1}^{n}\left|a_{j}\right|^{\theta_{j}}\right)
$$

for any $\theta_{j}>0, \sum_{j=1}^{n} \theta_{j}=1$, and $u_{j}, a_{j} \in D$. Next, (19) easily implies that the graph of $\Phi$ is convex.

Indeed, suppose that $\log a_{j} \in \Phi\left(u_{j}\right)$ for some functions $\log a_{j} \in D$ and $\log u_{j} \in D$, $1 \leq j \leq n$. We need to establish the same for their convex combinations $u$ and $a$,

$$
\begin{aligned}
\log u & =\sum_{j} \alpha_{j} \log u_{j}, \quad \alpha_{j} \geq 0, \quad \sum_{j} \alpha_{j}=1, \\
\log a & =\sum_{j} \alpha_{j} \log a_{j} .
\end{aligned}
$$

Since the sets $M\left(u_{j}, a_{j}\right)$ are nonempty, the set

$$
M\left(\prod_{j=1}^{n}\left|u_{j}\right|^{\alpha_{j}}, \prod_{j=1}^{n}\left|a_{j}\right|^{\alpha_{j}}\right)
$$

is also nonempty by (19). This means that

$$
\sum_{j=1}^{n} \alpha_{j} \log a_{j} \in \Phi\left(\sum_{j=1}^{n} \alpha_{j} \log u_{j}\right)
$$

as claimed.

Note that $D$ is a bounded and convex subset of $\mathrm{L}_{2}(w)$ for the weight $w=\left(M \chi_{B}\right)^{-\frac{1}{2}}$. Boundedness is a consequence of the following simple estimates for the function $\log u \in D$ :

$$
\int_{\{u \geq 1\}}\left[\left(M \chi_{B}\right) \log u\right]^{2} \leq \int_{\{u \geq 1\}} \frac{1}{\left(\frac{q}{2}\right)^{2}}\left[\log \left(u^{\frac{q}{2}}\right)\right]^{2} \leq\left(\frac{2}{q}\right)^{2} \int|u|^{q} \leq\left(\frac{2}{q}\right)^{2},
$$

because $M \chi_{B} \leq 1$ and $\log u \leq u$ for $u \geq 1$, and similarly,

$$
\int_{\{u<1\}}\left|\left(M \chi_{B}\right) \log u\right|^{2} \leq \int\left(\left[M \chi_{B}\right] \log \left[C_{q}^{-1} \delta M \chi_{B}\right]\right)^{2} \leq c^{\prime} \int\left(\left[M_{B}\right]^{\frac{2}{3}}\right)^{2} \leq c^{\prime \prime}
$$


for some constants $c^{\prime}$ and $c^{\prime \prime}$, because $u \geq C_{q}^{-1} M u \geq C_{q}^{-1} \delta M \chi_{B}$. Since the set $D$ is $(\nu \times \mu)$-closed, $D$ is also closed in $\mathrm{L}_{2}(w)$. We endow $D$ with the weak topology of $\mathrm{L}_{2}$, making it a convex, closed and compact subset of the locally convex linear topological space $\mathrm{L}_{2}(w)$ with the weak topology, which is metrizable on $D$.

We show that the graph of $\Phi$ is closed. Since the graph of $\Phi$ is convex, it suffices to show that it is closed in the strong topology of the space $\mathrm{L}_{2}(w)$. Suppose that we are given a sequence

$$
\log a_{j} \in \Phi\left(\log u_{j}\right)
$$

such that the sequences $\log a_{j} \in D$ and $\log u_{j} \in D$ converge in the space $\mathrm{L}_{2}(w)$ to some $\log A \in D$ and $\log U \in D$, respectively; we need to prove that $\log A \in \Phi(\log U)$. Passing to a subsequence, we may assume that the convergence in question is also almost everywhere. Again passing to a subsequence allows us to assume the rapid convergence

$$
\left\|\log a_{j}-\log A\right\|_{\mathrm{L}_{2}(w)} \leq 2^{-j} .
$$

Moreover, all $\log a_{j} \in D$ are bounded a.e. from below by a function finite a.e. because $a_{j} \geq C_{q}^{-1} M a_{j} \geq C_{q}^{-1} M\left(\chi_{B} \delta\right)$. Then the sequence

$$
\log \alpha_{j}=\bigvee_{k \geq j} \log a_{k} \geq \log a_{j}
$$

in $\mathrm{L}_{2}(w)$ is monotone decreasing and converges to $\log A$ almost everywhere. Similarly, the sequence

$$
\log \eta_{j}=\bigwedge_{k \geq j} \log v_{k} \leq \log v_{j}
$$

is monotone increasing and converges to $\log U$ almost everywhere. By the assumption (20), the sets $M\left(u_{j}, a_{j}\right)$ are nonempty, which means by (12) that the sets

$$
M\left(\eta_{j}, \alpha_{j}\right)
$$

are also nonempty and form a decreasing sequence because the sequences $f \eta_{j}$ and $\alpha_{j}^{-1}$ are monotone nondecreasing almost everywhere. These sets are nonempty, bounded in the lattice $X \mathrm{~L}_{q}$ having the Fatou property, and $(\nu \times \mu)$-bounded. An application of Theorem [6] to this sequence of sets shows that the set $M(U, A)$ is nonempty, whence $\log A \in \Phi(\log U)$. Thus, the graph of $\Phi$ is closed.

Applying the Ky Fan-Kakutani fixed point theorem (see 83 ) to the mapping $\Phi$, we conclude that there is some $\log u \in D$ such that $\log u \in \Phi(\log u)$. This means that the set $M(u, u)$ is nonempty. Suppose that $g \in M(u, u)$; then $g \in A_{p}$ with constant $C, g \geq|f| u$, and $\left\|g u^{-1}\right\|_{X} \leq m_{1}$. By the factorization of $A_{p}$ weights (see, e.g., [7, Chapter 5, 5.3]), there are $\omega_{0}, \omega_{1} \in A_{1}$ with a constant depending on $C$ only such that $g=\omega_{0} \omega_{1}^{1-p}$. By the same factorization theorems, $g u^{-1}=\omega_{0}\left(\omega_{1}^{\frac{p-1}{p}} u^{\frac{1}{p}}\right)^{-p}$ belongs to $\mathrm{A}_{p+1}$ with some constant $C_{1}$ depending only on $C$ and $C_{q}$, because, by Proposition [16, $\omega_{1}^{1-\frac{1}{p}} u^{\frac{1}{p}}$ belongs to $\mathrm{A}_{1}$ with a suitable estimate on the constant. Thus, $g u^{-1}$ is a suitable $\mathrm{A}_{p+1}$-majorant for $f$. The proof of Theorem 2 is complete.

\section{§5. BMO-REGULARITY FOR COUPLES OF LATTICES}

In this section we consider the BMO-regularity property for couples of lattices. We show that it is self-dual and stable under division by a lattice, and give another proof of Theorem 2. Although this proof involves additional notions, it might be even easier to understand in the technical aspect. 
Definition 4. A couple $(X, Y)$ of quasinormed lattices $X$ and $Y$ on $(S \times \Omega, \nu \times \mu)$ is said to be $\mathrm{A}_{p}$-regular with constants $(C, m)$ if for any $f \in X$ and $g \in Y, g \neq 0$, there exist majorants $u \in X, v \in Y, u \geq|f|, v \geq|g|$, such that $\|u\|_{X} \leq m\|f\|_{X},\|v\|_{Y} \leq m\|g\|_{X}$, and $\frac{u}{v} \in \mathrm{A}_{p}$ with constant $C$. A couple $(X, Y)$ is said to be BMO-regular with constant $(C, m)$ if for any $f \in X$ and $g \in Y, g \neq 0$, there exist majorants $u \in X, v \in Y, u \geq|f|$, $v \geq|g|$, such that $\|u\|_{X} \leq m\|f\|_{X},\|v\|_{Y} \leq m\|g\|_{X}$, and $\log \frac{u}{v} \in$ BMO with constant $C$. Such a couple of functions $(u, v)$ is called an $\mathrm{A}_{p}$-majorant or a BMO-majorant (respectively) for the couple $(f, g)$.

In the case of the unit circle $S=\mathbb{T}$, BMO-regularity for couples arises naturally in connection with interpolation of Hardy-type spaces of analytic functions (see, e.g., [1, 26]). In order to feel the flavor of these definitions, now we mention certain simple properties of BMO-regularity and $\mathrm{A}_{p}$-regularity for couples, which are mostly similar to the corresponding properties for a single lattice.

If lattices $X$ and $Y$ are BMO-regular, then the couple $(X, Y)$ is also BMO-regular, because we can merely take the BMO-majorants in the respective lattices to get a BMOmajorant for the couple. The converse is not true. For example, the couple $(X, X)$ is $\mathrm{A}_{1}$-regular for any lattice $X$, because for any functions $f$ and $g$ in $X$ we can take the BMO-majorants $u=\varphi\|f\|_{X}$ and $v=\varphi\|g\|_{X}, \varphi=\frac{f}{\|f\|_{X}} \vee \frac{g}{\|g\|_{X}}+h$, where $h \in X$ is any function of norm 1 positive a.e. (see Proposition 14). If lattices $X$ and $Y$ are $\mathrm{A}_{1}$-regular, then by factorization the couple $\left(X, Y^{p-1}\right)$ is $\mathrm{A}_{p}$-regular for $p>1$. If $X$ is $\mathrm{A}_{p}$-regular, then the couple $\left(X, \mathrm{~L}_{\infty}\right)$ is also $\mathrm{A}_{p}$-regular. If a couple $(X, Y)$ is BMO-regular or $\mathrm{A}_{2^{-}}$ regular, then the couple $(Y, X)$ is also BMO-regular or $\mathrm{A}_{2}$-regular (respectively), because $w \in \mathrm{A}_{2}$ with constant $C$ implies $w^{-1} \in \mathrm{A}_{2}$ with some constant depending only on $C$, and the relevant statement for $\log w \in \mathrm{BMO}$ is trivial. If $p>1$, then the $\mathrm{A}_{p}$-regularity of a couple implies its $\mathrm{A}_{q}$-regularity for some $q<p$. If a couple $(X, Y)$ is $\mathrm{A}_{p}$-regular,

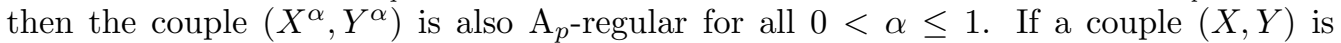
BMO-regular, then for any $p>1$ the couple $\left(X^{\alpha}, Y^{\alpha}\right)$ is $\mathrm{A}_{p}$-regular for all sufficiently small $\alpha$. If couples $(X, Y)$ and $(E, F)$ are BMO-regular, then the couple $(X E, Y F)$ is also BMO-regular.

As will be demonstrated in this section, BMO-regularity for a couple enjoys the same properties of self-duality and stability under division as BMO-regularity for a single lattice does. These results mostly follow [13, 26, where they were given in the case of the unit circle. First, we establish a characterization of $\mathrm{A}_{p}$-regularity for couples. For convenience we introduce the following notation:

$$
S_{X}^{+}=\left\{f \in X \mid\|f\|_{X}=1, \quad f>0 \text { a.e. }\right\}
$$

and

$$
S_{Y}^{+}=\left\{g \in Y \mid\|g\|_{Y}=1, \quad g>0 \text { a.e. }\right\} .
$$

Proposition 20. Suppose that $X$ and $Y$ are lattices on $(S \times \Omega, \nu \times \mu)$. The $\mathrm{A}_{p}$-regularity of the couple $(X, Y)$ with constants $(C, m)$ is equivalent to the following property: for all $f \in S_{X}^{+}$and $g \in S_{Y}^{+}$there exists $\alpha \in B \mathrm{~A}_{p}(C)$ such that

$$
\|\alpha g\|_{X} \leq c, \quad\left\|\alpha^{-1} f\right\|_{Y} \leq c
$$

with some constant $c$ independent of $f$ and $g$. The above constants $c$ and $m$ can be chosen to satisfy $c \leq m \leq c+1$.

Indeed, suppose that the couple $(X, Y)$ is $\mathrm{A}_{p}$-regular. Then for any $f \in S_{X}^{+}$and $g \in S_{Y}^{+}$ there exists an $\mathrm{A}_{p}$-majorant $(u, v)$, and the statement is true for $\alpha=\frac{u}{v}$. Conversely, suppose that the property claimed occurs and $f \in X, g \in Y, f \neq 0, g \neq 0$ are some functions. Making use of Proposition[14] and the invariance of the $\mathrm{A}_{p}$-regularity property 
under multiplication of $f$ and $g$ by some positive constants, we may assume that $f \in S_{X}^{+}$ and $g \in S_{Y}^{+}$. Let $u=\left(\frac{\alpha g}{f} \vee 1\right) f$ and $v=\left(\frac{f}{\alpha g} \vee 1\right) g$. Then $\|u\|_{X} \leq c+1$ and $\|v\|_{Y} \leq c+1$. It is easily seen that $\frac{u}{v}=\alpha \in B \mathrm{~A}_{p}(C)$, so $(u, v)$ is a suitable majorant.

Proposition 20 allows us to express the $A_{p}$-regularity property for couples in terms of the nonemptiness of certain sets that are closed, bounded, and convex. Specifically, suppose that $X$ and $Y$ are lattices with the Fatou property on $(S \times \Omega, \nu \times \mu)$. For $f \in X$, $g \in Y, f, g>0$ a.e., we introduce the set

$$
M_{C, c}^{(X, Y)}(f, g)=\left\{\alpha \mid\|\alpha g\|_{X} \leq c,\left\|\alpha^{-1} f\right\|_{Y} \leq c\right\} \cap B \mathrm{~A}_{p}(C) .
$$

Proposition 20 means that the existence of an $\mathrm{A}_{p}$-majorant with constants $(C, m)$ for functions $f$ and $g$ (that we may assume to be normalized) in the couple $(X, Y)$ is equivalent to the nonemptiness of the set (22) for some constant $c$ related to the constant $m$.

Let us highlight certain properties of the sets (22). The Fatou property and Proposition 16 ensure that $M_{C, c}(f, g)$ is convex and $(\nu \times \mu)$-closed (observe that $s \mapsto s^{-1}$ is a convex funtion for $s>0)$. It is easy to show that $g M_{C, c}^{(X, Y)}(f, g)$ is a convex, closed, and bounded subset of $X$. For any $f_{1} \geq|f|$ and $g_{1} \geq|g|$, we have

$$
M_{C, c}^{(X, Y)}(f, g) \supset M_{C, c}^{(X, Y)}\left(f_{1}, g_{1}\right) .
$$

Using the Hölder inequality for lattices (see (13)), it is easy to check that, like in (19), for any $\theta_{j}>0, \sum_{j=1}^{n} \theta_{j}=1$, and $f_{j} \in X, g_{j} \in Y$, we have

$$
\prod_{j=1}^{n}\left[M_{C, c}^{(X, Y)}\left(f_{j}, g_{j}\right)\right]^{\theta_{j}} \subset M_{C, c}^{(X, Y)}\left(\prod_{j=1}^{n}\left|f_{j}\right|^{\theta_{j}}, \prod_{j=1}^{n}\left|g_{j}\right|^{\theta_{j}}\right) .
$$

As before, on the left-hand part of (24) the multiplication and exponentiation are understood pointwise. In particular, substituting $f_{j}=f$ and $g_{j}=g$ in (24) yields the logarithmic convexity of the set $M_{C, c}^{(X, Y)}(f, g)$.

Proposition 21. Suppose that lattices $X$ and $Y$ on $(\mathbb{T} \times \Omega, m \times \mu)$ have the Fatou property and $f_{j} \in X, g_{j} \in Y$ are monotone nondecreasing sequences of functions that are positive a.e. and converge a.e. to some $f \in X$ and $g \in Y$. Then the intersection $\bigcap_{\gamma>0, j} M_{C, c+\gamma}^{(X, Y)}\left(f_{j}, g_{j}\right)$ coincides with the set $M_{C, c}^{(X, Y)}(f, g)$. If for all $\gamma>0$ and $j$ the sets $M_{C, c+\gamma}^{(X, Y)}\left(f_{j}, g_{j}\right)$ are nonempty, then the set $M_{C, c}^{(X, Y)}(f, g)$ is also nonempty.

The inclusion

$$
\bigcap_{\gamma>0, j} M_{C, c+\gamma}^{(X, Y)}\left(f_{j}, g_{j}\right) \supset M_{C, c}^{(X, Y)}(f, g)
$$

follows from (23). For any

$$
\alpha \in \bigcap_{\gamma>0, j} M_{C, c+\gamma}^{(X, Y)}\left(f_{j}, g_{j}\right)
$$

we have $\left\|\alpha g_{j}\right\|_{X} \leq c+\gamma$ and $\left\|\alpha^{-1} f_{j}\right\|_{Y} \leq c+\gamma$. Passing to the limit in these estimates and using of the Fatou property, we see that $\alpha \in M_{C, c}^{(X, Y)}(f, g)$. Finally, $F_{j}=g_{1} M_{C, c+\frac{1}{j}}^{(X, Y)}\left(f_{j}, g_{j}\right)$ is a decreasing sequence of sets that are closed in measure, convex, and bounded in $X$. Therefore, if these sets are nonempty, then by Theorem 6 their intersection $F=\bigcap_{j} F_{j}$ is also nonempty, which means that the set $M_{C, c}^{(X, Y)}(f, g)=g_{1}^{-1} F$ is nonempty. The proof of Proposition 21] is complete.

The $\mathrm{A}_{p}$-regularity property for couples admits the following somewhat more elegant version of Theorem [2. 
Theorem 7. Suppose that $X$ and $Y$ are Banach lattices with the Fatou property on $(S \times \Omega, \nu \times \mu)$. Suppose also that the lattices $X \mathrm{~L}_{q}$ and $Y \mathrm{~L}_{q}$ are Banach for some $1<q<$ $\infty$. If the couple $\left(X \mathrm{~L}_{q}, Y \mathrm{~L}_{q}\right)$ is $\mathrm{A}_{p}$-regular with constants $(C, m)$ for some $1 \leq p<\infty$, then the couple $(X, Y)$ is also $\mathrm{A}_{p}$-regular with the constants $(C, m+1)$.

As before, replacing $\mu$ by an equivalent measure we may assume that $\mu(\Omega)=1$. By Proposition 21, it suffices to prove that for any $f \in S_{X}^{+}$and $g \in S_{Y}^{+}$the set $M_{C, c}^{(X, Y)}(f, g)$ is nonempty. Next, by Proposition 21 it suffices to prove that for any number $\gamma>0$ and any ball $B_{0}$ of $S$ the set $M_{C, c+\gamma}^{(X, Y)}\left(f \chi_{B}, g \chi_{B}\right)$ is nonempty, where $B=B_{0} \times \Omega$ as before.

This time we introduce the set $D=\left\{\log h \mid\|h\|_{\mathrm{L}_{q}(B)} \leq 1, h \geq \delta\right\}$ of measurable functions supported on $B$ for $0<\delta<\nu(B)^{-\frac{1}{q}}$. The set $D$ is nonempty, closed in measure, and convex due to the Hölder inequality. Since the couple $\left(X \mathrm{~L}_{q}, Y \mathrm{~L}_{q}\right)$ is $\mathrm{A}_{p}$-regular, Proposition 20 shows that for any $(\log u, \log v) \in D \oplus D$ the set $M_{C, c}^{\left(X \mathrm{~L}_{q}, Y \mathrm{~L}_{q}\right)}(u f, v g)$ is nonempty. Using estimate (17), we see that if

$$
(\log u, \log v) \in D \oplus D
$$

and

then

$$
\alpha \in M_{C, c}^{\left(X \mathrm{~L}_{q}, Y \mathrm{~L}_{q}\right)}(u f, v g)
$$

and

$$
c \geq\|\alpha v g\|_{X \mathrm{~L}_{q}} \geq\left(1+\delta \nu(B)^{\frac{1}{q}}\right)^{-1} \inf _{\log a \in D}\left\|\alpha g v a^{-1}\right\|_{X}
$$

$$
c \geq\left\|\alpha^{-1} u f\right\|_{Y \mathrm{~L}_{p}} \geq\left(1+\delta \nu(B)^{\frac{1}{q}}\right)^{-1} \inf _{\log b \in D}\left\|\alpha^{-1} f u b^{-1}\right\|_{Y} .
$$

Fix some $\delta$ such that $c\left(1+\delta \nu(B)^{\frac{1}{q}}\right) \leq c+\gamma$. Then estimates (25) and (26) imply that for all $(\log u, \log v) \in D \oplus D$ the set

$$
\Phi(\log u, \log v)=\left\{(\log a, \log b) \in D \oplus D \mid M_{C, c+\gamma}^{(X, Y)}\left(f u a^{-1}, g v b^{-1}\right) \neq \varnothing\right\}
$$

is nonempty. Employing (24), it is easy to show that, as before, the graph of $\Phi$ is convex. Now, $D$ is a bounded and convex subset of $\mathrm{L}_{2}$. Since $D$ is closed in measure, $D$ is also closed in $\mathrm{L}_{2}$. Endow $D \oplus D$ with the weak topology of the space $\mathrm{L}_{2} \oplus \mathrm{L}_{2}$; then $D \oplus D$ becomes a convex, closed, and compact set in $\mathrm{L}_{2} \oplus \mathrm{L}_{2}$. By the Fatou property, the set $\Phi(\log u, \log v)$ is closed in measure for any $(\log u, \log v) \in D \oplus D$, so it is closed in the strong topology of $\mathrm{L}_{2} \oplus \mathrm{L}_{2}$, and therefore in $D \oplus D$, because of convexity.

The closedness of the graph of $\Phi$ is verified as before. Since the graph is a convex set, it suffices to prove that it is closed in the strong topology of the space $\mathrm{L}_{2} \oplus \mathrm{L}_{2}$. Suppose that

$$
\left(\log a_{j}, \log b_{j}\right) \in \Phi\left(\log u_{j}, \log v_{j}\right)
$$

for some sequences of functions $\log a_{j} \in D, \log b_{j} \in D, \log u_{j} \in D, \log v_{j} \in D$ converging in $\mathrm{L}_{2}$ to some $\log A \in D, \log B \in D, \log U \in D$, and $\log V \in D$, respectively. We need to prove that

$$
(\log A, \log B) \in \Phi(\log U, \log V) .
$$

By passing to a subsequence, we may assume that the above convergence is almost everywhere. Passing to a subsequence again, we can assume that the rapid convergence

$$
\begin{aligned}
\left\|\log a_{j}-\log A\right\|_{\mathrm{L}_{2}} & \leq 2^{-j}, \\
\left\|\log b_{j}-\log B\right\|_{\mathrm{L}_{2}} & \leq 2^{-j}
\end{aligned}
$$


occurs. All these functions are bounded from below by $\log \delta$. Then the sequences

$$
\begin{aligned}
& \log \alpha_{j}=\bigvee_{k \geq j} \log a_{k} \geq \log a_{j}, \\
& \log \beta_{j}=\bigvee_{k \geq j} \log b_{k} \geq \log b_{j}
\end{aligned}
$$

in $\mathrm{L}_{2}$ are monotone nonincreasing and converge to $\log A$ and $\log B$ almost everywhere. Similarly, the sequences

$$
\begin{aligned}
& \log \eta_{j}=\bigwedge_{k \geq j} \log u_{k} \leq \log u_{j}, \\
& \log \zeta_{j}=\bigwedge_{k \geq j} \log v_{k} \leq \log v_{j}
\end{aligned}
$$

are monotone nondecreasing and converge to $\log U$ and $\log V$ almost everywhere. By the assumption (27), the sets $M_{C, c+\gamma}^{(X, Y)}\left(f u_{j} a_{j}^{-1}, g v_{j} b_{j}^{-1}\right)$ are nonempty; therefore, by (23) the sets

$$
M_{C, c+\gamma}^{(X, Y)}\left(f \eta_{j} \alpha_{j}^{-1}, g \zeta_{j} \beta_{j}^{-1}\right)
$$

are also nonempty and form a decreasing sequence, because the sequences $f \eta_{j} \alpha_{j}^{-1}$ and $g \zeta_{j} \beta_{j}^{-1}$ are monotone nondecreasing almost everywhere. An application of Proposition 21 to this sequence of sets shows that the set $M_{C, c+\gamma}^{(X, Y)}\left(f U A^{-1}, g V B^{-1}\right)$ is nonempty. Thus, $(\log A, \log B) \in \Phi(\log U, \log V)$ as claimed, and the graph of $\Phi$ is closed.

Finally, we apply the Ky Fan-Kakutani theorem to the mapping $\Phi$ and conclude that there exists some $(\log u, \log v) \in D \oplus D$ such that

$$
(\log u, \log v) \in \Phi(\log u, \log v) .
$$

This means that the set

$$
M_{C, c+\gamma}^{(X, Y)}\left(f \chi_{B}, g \chi_{B}\right)=M_{C, c+\gamma}^{(X, Y)}\left(f u u^{-1}, g v v^{-1}\right)
$$

is nonempty as claimed. The proof of Theorem 7 is complete.

Proposition 22. Suppose that $X, Y, E$, and $F$ are Banach lattices on $(S \times \Omega, \nu \times \mu)$. If the couple $(X, Y)$ is $\mathrm{A}_{p}$-regular, then the couple $(X F, Y F)$ is also $\mathrm{A}_{p}$-regular. The same is true for $\mathrm{BMO}$-regularity. If the couples $(X, Y)$ and $(E, F)$ are $\mathrm{BMO}$-regular, then the couple $(X E, Y F)$ is also BMO-regular.

Indeed, if $f \in X F$ and $g \in Y F, g \neq 0$, then the definition of the lattice product shows that there exist decompositions $f=a \varphi_{0}$ and $g=b \varphi_{1}$ such that $a \in X, b \in Y$, $0 \leq \varphi_{0} \in F, 0 \leq \varphi_{1} \in F,\left\|\varphi_{0}\right\|_{F} \leq 1$, and $\left\|\varphi_{1}\right\|_{F} \leq 1$. For $a$ and $b$ we can find the corresponding majorant $(u, v)$ in the couple $(X, Y)$. Let $\varphi=\varphi_{0} \vee \varphi_{1}$. Then $(u \varphi, v \varphi)$ is a suitable majorant for the functions $f$ and $g$ in the couple $(X F, Y F)$, because $\|\varphi\|_{F} \leq 2$. The other claims of Proposition 22 are trivial.

Proposition 23. Suppose that $X$ is a quasinormed lattice on $(S \times \Omega, \nu \times \mu)$. If the couple $\left(X, \mathrm{~L}_{\infty}\right)$ is either $\mathrm{A}_{p}$-regular or $\mathrm{BMO}$-regular, then the lattice $X$ is also $\mathrm{A}_{p}$-regular or BMO-regular, respectively.

In order to prove Proposition 23, it suffices to observe that if $f \in X$ and $(u, v)$ is a suitable majorant in the couple $\left(X, \mathrm{~L}_{\infty}\right)$ for $(f, 1)$, then $1 \leq v \leq m$ almost everywhere. Therefore, $u=\frac{u}{v} \cdot v$ belongs to $\mathrm{A}_{p}$, or $\log u$ belongs to BMO, respectively, with suitable estimates for the constant, whence $u$ is a suitable majorant for $f$.

The following proposition follows from the remarks after Definition 4 and the argument near the end of 4 . 
Proposition 24. If a lattice $Y$ is $\mathrm{A}_{p}$-regular and a lattice $Z$ is $\mathrm{A}_{1}$-regular, then the couple $(Y, Z)$ is $\mathrm{A}_{p+1}$-regular.

Indeed, suppose we are given $y \in Y$ and $z \in Z$. Let $a$ and $b$ be some of their respective $\mathrm{A}_{p}$-majorants and $\mathrm{A}_{1}$-majorants. Using factorization of $\mathrm{A}_{p}$ weights (see, e.g., [7. Chapter 5, 5.3]), we can write $a=w_{0} w_{1}^{1-p}$ for some $w_{0}, w_{1} \in \mathrm{A}_{1}$ with suitable estimates on the constants. Then

$$
\frac{a}{b}=\frac{w_{0}}{w_{1}^{p-1} b}=\frac{w_{0}}{\left[w_{0}^{1-\frac{1}{p}} b^{\frac{1}{p}}\right]^{p}} \in \mathrm{A}_{p+1},
$$

because $w_{0}^{1-\frac{1}{p}} b^{\frac{1}{p}} \in \mathrm{A}_{1}$ by Proposition [16, so that the couple $(a, b)$ is a suitable $\mathrm{A}_{p+1^{-}}$ majorant for $y$ and $z$.

Now we can give another proof of Theorem 2, If under its conditions the lattice $X \mathrm{~L}_{q}$ is $\mathrm{A}_{p}$-regular, then, by Proposition 24, the couple $\left(X \mathrm{~L}_{q}, \mathrm{~L}_{q}\right)$ is $\mathrm{A}_{p+1}$-regular, because the lattice $\mathrm{L}_{q}$ is $\mathrm{A}_{1}$-regular by Proposition 2. Application of Theorem 7 to this couple yields the $\mathrm{A}_{p+1}$-regularity of the couple $\left(X, \mathrm{~L}_{\infty}\right)$. Therefore, by Proposition 23 , the lattice $X$ is $\mathrm{A}_{p+1}$-regular as claimed, which concludes the alternative proof of Theorem 2

Now we state the main result of this section.

Theorem 8. Suppose that $X$ and $Y$ are Banach lattices with the Fatou property on $(S \times \Omega, \nu \times \mu)$. The following conditions are equivalent.

1) $(X, Y)$ is BMO-regular.

2) $\left(X \mathrm{~L}_{q}, Y \mathrm{~L}_{q}\right)$ is BMO-regular for some (equivalently, for all) $0<q \leq \infty$.

3) $\left(X^{\prime}, Y^{\prime}\right)$ is BMO-regular.

4) $X Y^{\prime}$ is BMO-regular.

Indeed, the implication $1 \Rightarrow 2$ follows from Proposition 22 The implication $2 \Rightarrow 1$ follows from Theorem 7 applied to the couple $\left(\left[X \mathrm{~L}_{q}\right]^{\delta},\left[Y \mathrm{~L}_{q}\right]^{\delta}\right)$ for sufficiently small values of $\delta$. We verify that $1 \Rightarrow 3$. If a couple $(X, Y)$ is BMO-regular, then the couple $\left(X^{\frac{1}{2}}, Y^{\frac{1}{2}}\right)$ is also BMO-regular. Applying Proposition 22 with $F=X^{\prime \frac{1}{2}} Y^{\prime \frac{1}{2}}$, we see that the couple

$$
\left(X^{\frac{1}{2}}\left(X^{\prime \frac{1}{2}} Y^{\prime \frac{1}{2}}\right), Y^{\frac{1}{2}}\left(X^{\prime \frac{1}{2}} Y^{\prime \frac{1}{2}}\right)\right)=\left(\left(X X^{\prime}\right)^{\frac{1}{2}} Y^{\prime \frac{1}{2}},\left(Y Y^{\prime}\right)^{\frac{1}{2}} X^{\prime \frac{1}{2}}\right)=\left(Y^{\prime \frac{1}{2}} \mathrm{~L}_{2}, X^{\prime \frac{1}{2}} \mathrm{~L}_{2}\right)
$$

is also BMO-regular. Therefore, by the already established implication $2 \Rightarrow 1$, the couple $\left(Y^{\prime \frac{1}{2}}, X^{\prime \frac{1}{2}}\right)$ is BMO-regular, so the couple $\left(X^{\prime}, Y^{\prime}\right)$ is BMO-regular, i.e., condition 3 holds true. Because of the Fatou property, the couples $(X, Y)$ and $\left(X^{\prime}, Y^{\prime}\right)$ can be interchanged in the statement of the theorem; therefore, by the implications already established, conditions 1 and 3 are equivalent. Thus, we have proved that conditions 1-3 are equivalent. Before we show that condition 4 is equivalent to the other conditions, we make a few simple remarks.

Theorem 8 expresses the properties of BMO-regularity for couples that can be conveniently referred to as self-duality and stability under division by a lattice. In fact, by the stability under division it is more natural to mean the following property, which is a simple consequence of the implication $2 \Rightarrow 1$ in Theorem 8 .

Proposition 25. Suppose that $X, Y, E$, and $F$ are Banach lattices with the Fatou property on $(S \times \Omega, \nu \times \mu)$. If the couples $(X E, Y F)$ and $(E, F)$ are BMO-regular, then the couple $(X, Y)$ is also BMO-regular.

This proposition is converse to Proposition 22 in a certain sense. For the proof it suffices to observe that the couple $\left(E^{\prime}, F^{\prime}\right)$ is BMO-regular by the implication $1 \Rightarrow 3$ in Theorem 8 the couple $\left(X E E^{\prime}, Y F F^{\prime}\right)=\left(X \mathrm{~L}_{1}, Y \mathrm{~L}_{1}\right)$ is BMO-regular by Proposition 22 . The claim then follows by yet another use of the implication $2 \Rightarrow 1$ in Theorem 8 . 
Proposition 26. Suppose that $X$ and $Y$ are Banach lattices with Fatou property on $(S \times \Omega, \nu \times \mu)$. If $Y$ and $(X, Y)$ are both BMO-regular, then $X$ is also BMO-regular.

Indeed, under the assumptions of Proposition 26, the couple $(E, F)=\left(\mathrm{L}_{\infty}, Y\right)$ is BMO-regular, and therefore, by Proposition 25, the couple $\left(X, \mathrm{~L}_{\infty}\right)$ is BMO-regular; by Proposition 23 this means that $X$ is BMO-regular. Here we mention that the transition to BMO-regularity of the lattice $X Y$ in Proposition 26 can be made constructively, but perhaps not as easily as the argument presented here; see [13, Lemma 1].

Finally, we prove that condition 4 in Theorem 8 is equivalent to the other conditions. If condition 1 is satisfied, then by Proposition 22, the couple $\left(X Y^{\prime}, Y Y^{\prime}\right)=\left(X Y^{\prime}, \mathrm{L}_{1}\right)$ is BMO-regular, and by Proposition 26 the lattice $X Y^{\prime}$ is BMO-regular, i.e., condition 4 is satisfied. If condition 4 is satisfied, then the couple $\left(\mathrm{L}_{1}, X Y^{\prime}\right)$ is BMO-regular. Multiplying it by $X^{\prime}$ and using Proposition 22, we see that the couple

$$
\left(\mathrm{L}_{1} X^{\prime},\left(X Y^{\prime}\right) X^{\prime}\right)=\left(\mathrm{L}_{1} X^{\prime}, Y^{\prime}\left(X X^{\prime}\right)\right)=\left(X^{\prime} \mathrm{L}_{1}, Y^{\prime} \mathrm{L}_{1}\right)
$$

is BMO-regular, i.e., condition 2 is satisfied for the lattices $X^{\prime}$ and $Y^{\prime}$. The proof of Theorem 8 is complete.

\section{§6. Proof of Theorem 4}

The proof of Theorem 4 presented in this section is merely a slight refinement of the proof of Theorem 3.5 in [1, which is in turn a variant of the well-known Maurey-Krivine factorization theorem (see [9]). For the first time, in a similar context these ideas were exploited in [46. Suppose that a lattice $Y$ and an operator $T$ satisfy the assumptions of Theorem 4 and we are given some $f \in Y^{\prime}$; we need to find a function $w$ such that $w \geq|f|,\|w\|_{Y^{\prime}} \leq c_{0}\|f\|_{Y^{\prime}}$, and $\|T\|_{L_{2}\left(w^{-\frac{1}{2}}\right)} \leq c_{1}$ with suitable constants $c_{0}$ and $c_{1}$. We may assume without loss of generality that $\|f\|_{Y^{\prime}}=1$ and $f \in Y_{+}^{\prime}$. Moreover, Proposition 14 allows us to assume that $|f|>0$ almost everywhere. First, observe that for $g \in Y^{\frac{1}{2}}$ and all $y \in Y_{+}^{\prime}$ we have

$$
\int|T g|^{2}|y| \leq\|T g\|_{Y^{\frac{1}{2}}}^{2}\|y\|_{Y^{\prime}} \leq \rho^{2}\|y\|_{Y^{\prime}}\|g\|_{Y^{\frac{1}{2}}}^{2}
$$

where $\rho=\|T\|_{Y^{\frac{1}{2}}}$, i.e., $\|T\|_{Y^{\frac{1}{2}} \rightarrow \mathrm{L}_{2}\left(y^{-\frac{1}{2}}\right)} \leq \rho$. Let $a>\rho K_{G}$ be a number as in the assumptions of the theorem, $K_{G}$ being the Grothendieck constant (see §3). Suppose that $F_{1}$ is the open unit ball of the lattice $Y$ centered at the origin, and put

$$
\begin{aligned}
& F_{2}=\operatorname{co}\{u \in Y \mid u>0 \text { a.e. and } \\
& \left.\qquad\|T v\|_{\mathrm{L}_{2}\left(y^{-\frac{1}{2}}\right)} \geq a \text { for some } v \in Y^{\frac{1}{2}} \text { such that }|v| \leq u^{\frac{1}{2}}\right\} .
\end{aligned}
$$

By co we denote the convex hull of a set. We only consider functions $y \in Y_{+}^{\prime}$ such that $y>0$ a.e. and $\|y\|_{Y^{\prime}} \leq 1$. Thus, if $\|T\|_{Y^{\frac{1}{2}} \rightarrow \mathrm{L}_{2}\left(y^{-\frac{1}{2}}\right)}=0$ for some $y$, then $T g=0$ for any $g \in Y^{\frac{1}{2}}$, because, by the assumptions, $\operatorname{supp} Y=S \times \Omega$ and $Y^{\frac{1}{2}} \cap \mathrm{L}_{2}\left((y \vee|f|)^{-\frac{1}{2}}\right)$ is dense in $\mathrm{L}_{2}\left((y \vee|f|)^{-\frac{1}{2}}\right)$ (see Proposition 14), whence $\|T\|_{\mathrm{L}_{2}\left((y \vee|f|)^{-\frac{1}{2}}\right)}=0$, and $y \vee|f|$ is a suitable majorant. Thus, we may assume without loss of generality that $\|T\|_{Y^{\frac{1}{2}} \rightarrow \mathrm{L}_{2}\left(y^{-\frac{1}{2}}\right)}>0$, which means that $F_{2}$ is nonempty. We show that the sets $F_{1}$ and $F_{2}$ have empty intersection.

Indeed, suppose that there exists $g \in F_{1} \cap F_{2}$. Then $g \in Y, g>0$ a.e., $\|g\|_{Y} \leq 1$, and for some functions $u_{j} \in Y$ positive a.e., $1 \leq j \leq n$, and appropriate functions $v_{j}$, $\left|v_{j}\right| \leq u_{j}^{\frac{1}{2}}$ for all $1 \leq j \leq n$, and numbers $\alpha_{j}>0,1 \leq j \leq n, \sum_{j=1}^{n} \alpha_{j}=1$, we have 
$g=\sum_{j=1}^{n} \alpha_{j} u_{j}$. Using (28) and the Grothendieck theorem (see 93 ), we can carry out the following estimate:

$$
\begin{aligned}
1 \geq\|g\|_{Y} & =\left\|\left(\sum_{j=1}^{n} \alpha_{j} u_{j}\right)^{\frac{1}{2}}\right\|_{Y^{\frac{1}{2}}} \geq\left\|\left(\sum_{j=1}^{n}\left|\alpha_{j}^{\frac{1}{2}} v_{j}\right|^{2}\right)^{\frac{1}{2}}\right\|_{Y^{\frac{1}{2}}} \\
& \geq \frac{1}{K_{G}}\left\|\left(\sum_{j=1}^{n}\left|\alpha_{j}^{\frac{1}{2}} T v_{j}\right|^{2}\right)^{\frac{1}{2}}\right\|_{Y^{\frac{1}{2}}} \geq \frac{1}{\rho K_{G}}\left\|\left(\sum_{j=1}^{n}\left|\alpha_{j}^{\frac{1}{2}} T v_{j}\right|^{2}\right)^{\frac{1}{2}}\right\|_{\mathrm{L}_{2}\left(y^{-\frac{1}{2}}\right)} \\
& =\frac{1}{\rho K_{G}}\left(\sum_{j=1}^{n} \alpha_{j}\left\|T v_{j}\right\|_{\mathrm{L}_{2}\left(y^{-\frac{1}{2}}\right)}^{2}\right)^{\frac{1}{2}} \geq \frac{a}{\rho K_{G}}>1,
\end{aligned}
$$

a contradiction. Thus, $F_{1} \cap F_{2}=\varnothing$. The sets $F_{1}$ and $F_{2}$ are subsets of the Banach space $Y$. They are nonempty and convex, and $F_{1}$ is open. Therefore, there exists a continuous functional $z_{y}$ in $Y^{*}$ separating these sets. By assumption, we have $Y^{*}=Y^{\prime}$, whence $z_{y} \in Y^{\prime}$. Choosing a suitable normalization for $z_{y}$ yields

$$
\sup \left\{\operatorname{Re} \int u z_{y} \mid u \in F_{1}\right\} \leq 1 \leq \inf \left\{\operatorname{Re} \int u z_{y} \mid u \in F_{2}\right\} .
$$

Since $F_{1}$ is the unit ball of $Y$ and $F_{2}$ lies in the cone of the functions nonnegative a.e., we may assume that $z_{y}$ is nonnegative almost everywhere. From the first inequality in (29) we see that $\left\|z_{y}\right\|_{Y^{\prime}} \leq 1$. The second inequality in (29) means that for any $v \in Y^{\frac{1}{2}}$ with $T v \neq 0$ in the space $\mathrm{L}_{2}\left(y^{-\frac{1}{2}}\right)$ (whence

$$
\left.\frac{a^{2}|v|^{2}}{\|T v\|_{\mathrm{L}_{2}\left(y^{-\frac{1}{2}}\right)}^{2}} \in F_{2}\right),
$$

we have

$$
1 \leq \int \frac{a^{2}|v|^{2}}{\|T v\|_{\mathrm{L}_{2}\left(y^{-\frac{1}{2}}\right)}^{2}} z_{y},
$$

which means that

$$
\int|T v|^{2} y \leq a^{2} \int|v|^{2} z_{y}
$$

for all $v \in Y^{\frac{1}{2}}$. Since the norm of a weighted $\mathrm{L}_{2}$ lattice is order continuous and $\operatorname{supp} Y=$ $S \times \Omega$, it is easy to extend estimate (30) to all $v \in \mathrm{L}_{2}\left(y^{-\frac{1}{2}}\right)$ (see Proposition 14); thus, $\|T\|_{\mathrm{L}_{2}\left(y^{-\frac{1}{2}}\right) \rightarrow \mathrm{L}_{2}\left(z_{y}^{-\frac{1}{2}}\right)} \leq a$. Now we build inductively a sequence $y_{0}=f, y_{j}=z_{y_{j-1}}$ for $j \in \mathbb{N}$, and put $w=\sum_{j \geq 0} m^{-j} y_{j}$ for some $m>1$. Then

$$
\|w\|_{Y^{\prime}} \leq \sum_{j \geq 0} m^{-j}\|f\|_{Y^{\prime}}=\frac{m}{m-1}
$$

and

$$
\|T f\|_{\mathrm{L}_{2}\left(w^{-\frac{1}{2}}\right)}^{2} \leq a^{2} m\|f\|_{\mathrm{L}_{2}\left(w^{-\frac{1}{2}}\right)}^{2}, \quad f \in \mathrm{L}_{2}\left(w^{-\frac{1}{2}}\right) .
$$

Thus, $w$ is a suitable majorant, which concludes the proof of Theorem 4 


\section{§7. CONCLUding REMARKS}

In this section we give various remarks along with a (perforce) brief and incomplete account of the history of the theory.

As it was mentioned in the Introduction, the notion of BMO-regularity was, apparently for the first time, introduced explicitly in 22 in connection with interpolation of Hardytype spaces on the unit circle $\mathbb{T}$; see the survey [1] and also [22, 12, 26]. The theory of BMO-regularity on the unit circle is somewhat richer, because the condition log $w \in \mathrm{BMO}$ has a lot of useful characterizations in terms of analytic functions; however, in the present paper we have shown that, as it usually happens in harmonic analysis, all fundamental results dealing with the notion of BMO-regularity itself are in fact completely within the realm of the real analysis.

The classical doubling property in the space of homogeneous type $\left(\mathbb{R}^{n}, \nu\right)$ (or even in a general space $(S, \nu))$ is, apparently, not necessary for most of the results of this paper, so that it can be dropped or relaxed; see, e.g., 45] on relaxing the doubling property.

Our definition of BMO-regularity (see Definition 1) differs formally from the one used in the cited works only in the following: we do not impose the restriction $f \neq 0$ in the majorization property. This suits our technique better, because when dealing with $\mathrm{A}_{p}$-regularity it is natural to assume that $0 \in \mathrm{A}_{p}$. We do not restrict the weights to functions positive a.e.; however, it is easily seen that if $\log w \in \mathrm{BMO}$, then for almost all $\omega \in \Omega$ the function $w(\cdot, \omega)$ is either zero a.e., or positive a.e., and the same is true for $\mathrm{A}_{p}$ weights, as they also satisfy $\log w \in$ BMO.

BMO-regularity for rearrangement invariant Banach lattices on the circle, i.e., Proposition 2 for this case, was essentially established in 36, where a majorant with a corresponding analytic decomposition of unity was constructed explicitly; see also 3 and the more recent paper [14, where it was proved that the existence of such a majorant is equivalent to BMO-regularity. It is unclear to what extent this symmetry condition can be relaxed. In particular, it is unclear if in the case of $\mathbb{R}^{n}$ or $\mathbb{T}$ all translation invariant lattices, i.e., those that have the property $\|f(\cdot+h)\|=\|f\|$ for arbitrary $f$ and $h$, are BMO-regular.

Many results concerning BMO-regularity in a slightly more general context, although with various additional and sometimes heavy restrictions on the lattices, originate from 22], where they were established via complex interpolation of Hardy-type spaces. For the case of BMO-regularity on the unit circle $\mathbb{T}$ and the operator of harmonic conjugation $T=$ $\mathcal{H}$, all results of the present paper have been known for some time, see [1, 12, 13, 25, 26]. However, in the previous work the stability of BMO-regularity under duality and division by a BMO-regular lattice, which is arguably the most interesting point in the theory, was based on the real interpolation of Hardy-type spaces and the characterization of BMO-regularity in terms of the analytic decomposition of unity. The present paper shows that all results pertaining to BMO-regularity itself can be obtained with less effort and without any reference to interpolation or analytic functions. Note, however, that the Fatou property is essential to our key arguments, whereas in some of the previous work there are some results where it can be spared by other properties like the order continuity.

The main auxiliary result of this paper, which is Theorem 2, can be reformulated in terms introduced in [13]: for lattices with the Fatou property the weak BMO-regularity coincides with the usual one. A lattice $X$ is said to be weakly BMO-regular if $X \mathrm{~L}_{q}$ is BMO-regular for some $q$. For the weak BMO-regularity on $\mathbb{R}^{n}$ or $\mathbb{T}$ it is easy to obtain the same main results (see Theorem B in the Introduction) without the Fatou property (and without Theorem 2), because self-duality can be established by using characterization in terms of boundedness of the Riesz (or Hilbert) transformations. This 
approach makes it possible to dispense of the Grothendieck theorem as well in the case of the unit circle, because the boundedness of the Hilbert transformation together with some simple complex analysis easily yield BMO-regularity (see [1, Lemma 3.1]). The weak BMO-regularity, however, is a less informative property, as it is harder to apply it to interpolation (see [13]), and the weak BMO-regularity of $\mathrm{L}_{\infty}\left(\ell^{1}\right)$ follows directly from Proposition 1 .

The convexity and closedness in measure of the set of BMO-majorants under appropriate conditions, which we rely on, is not quite trivial, see [26, §2] for a more detailed exposition. However, working directly with $\mathrm{A}_{p}$-regularity reduces the difficulties considerably (see Proposition 16). For example, logarithmic convexity is obtained without complex interpolation, which would require that the corresponding operator in the definition of the regularity be linear (see (9)).

The notion of BMO-regularity for couples arises naturally in the interpolation theory for Hardy-type spaces on the unit circle, and, at least in a number of cases, characterizes "good" interpolation. It is not clear what applications it might have in the general case.

Note that the theory of BMO-regularity presented here can apparently be generalized directly to a large extent to the case of the Calderón-Zygmund singular integral operators in several variables, i.e., to the operators defined in the space $\mathrm{L}_{2}\left(\prod_{j=1}^{m}\left(S_{j}, \nu_{j}\right)\right)$ that are Calderón-Zygmund in each variable; here $\left(S_{j}, \nu_{j}\right)$ are some spaces of homogeneous type. The orthogonal projection $\mathbb{P}$ onto $\mathrm{H}_{2}\left(\mathbb{T}^{n}\right)$ in $\mathrm{L}_{2}\left(\mathbb{T}^{n}\right)$ is a prominent example of such an operator.

Proposition 13] is a version of a well-known extrapolation technique; the proper bibliography and application to spaces $\mathrm{L}_{p(\cdot)}$ for a large number of examples of various "classical" mappings and vector-valued estimates can be found in [29. Many modular lattices, which usually have the Fatou property, can be represented as $\left(X^{\prime}\right)^{\delta}$, so that the boundedness of singular operators can be reduced to the $\mathrm{A}_{p}$-regularity of the corresponding lattice $X$.

In conclusion, let us point out briefly that $A_{1}$-regularity can be applied to the real interpolation of spaces that can be defined in terms of Calderón-Zygmund singular integral operators, in particular, the real classes $\mathrm{H}_{p}$ (see, e.g., [1, 38]). Suppose that $P$ is a Calderón-Zygmund projection with estimate of the form (3) on its kernel and the kernel of the adjoint operator, which is defined on $\mathrm{L}_{s}$ for some (and therefore for all) $1<s<\infty$. Then for a lattice $X$, we can define the space

$$
X^{P}=\{f \in X \mid P f=f\}
$$

if we extend $P$ to $X$ somehow; this may pose some difficulties. It is convenient to denote $\left[\mathrm{L}_{p}(w)\right]^{P}$ by $\mathcal{H}_{p}^{P}(w)$. The results of [37] can be used to show that the couple $\left(\mathcal{H}_{1}^{P}\left(\omega_{0}^{-1}\right), \mathcal{H}_{\infty}^{P}\left(\omega_{1}\right)\right)$ is $K$-closed in $\left(\mathrm{L}_{1}\left(\omega_{0}^{-1}\right), \mathrm{L}_{\infty}\left(\omega_{1}\right)\right)$ for two weights $\omega_{0}, \omega_{1} \in \mathrm{A}_{1}$ such that $\omega_{0} \omega_{1} \in \mathrm{A}_{\infty}$ with a proper estimate for the constant of $K$-closedness. This means that if $X$ and $Y$ are $\mathrm{A}_{1}$-regular lattices with a certain condition on the constants, then the couple $\left(X^{\prime P}, Y^{P}\right)$ is $K$-closed 7 in $\left(X^{\prime}, Y\right)$.

\section{ACKNOWLEDGEMENT}

The author is grateful to his advisor S. V. Kislyakov for numerous remarks and suggestions to early versions of this paper.

\footnotetext{
${ }^{7}$ Note added in translation. This application to interpolation was explored in detail (to some extent) in the author's thesis 27 .
} 


\section{REFERENCES}

[1] S. V. Kisliakov, Interpolation of $H_{p}$-spaces: some recent developments, Function Spaces, Interpolation Spaces, and Related Topics (Haifa, 1995), Israel Math. Conf. Proc., vol. 13, Bar-Ilan Univ., Ramat Gan, 1999, pp. 102-140. MR.1707360 (2000f:46028)

[2] L. Diening, P. Hästö, and A. Nekvinda, Open problems in variable exponent Lebesgue and Sobolev spaces, FSDONA 2004 Proceedings (Drabek and Rakosnik, eds.), Milovy, Czech Republic, 2004, pp. $38-58$.

[3] S. V. Kisliakov and Quan Hua Xu, Interpolation of weighted and vector-valued Hardy spaces, Trans. Amer. Math. Soc. 343 (1994), no. 1, 1-34. MR.1236225 (95b:46038)

[4] - Partial retractions for weighted Hardy spaces, Studia Math. 138 (2000), no. 3, 251-264. MR.1758858 (2001i:46031)

[5] M. Cwikel, J. E. McCarthy, and T. H. Wolff, Interpolation between weighted Hardy spaces, Proc. Amer. Math. Soc. 116 (1992), no. 2, 381-388. MR1093595 (92m:46033)

[6] J. B. Garnett, Bounded analytic functions, Pure Appl. Math., vol. 96, Acad. Press, New YorkLondon, 1981. MR0628971 (83g:30037)

[7] E. M. Stein, Harmonic analysis: real-variable methods, orthogonality, and oscillatory integrals, Princeton Math. Ser., vol. 43, Princeton Univ. Press, Princeton, NJ, 1993. MR1232192 (95c:42002)

[8] Donggao Deng and Yongsheng Han, Harmonic analysis on spaces of homogeneous type, Lecture Notes in Math., vol. 1966, Springer-Verlag, Berlin, 2009. MR 2467074 (2010i:43005)

[9] J. Lindenstrauss and L. Tzafriri, Classical Banach spaces. I, II, Ergeb. Math. Grenzgeb., Bd. 92, 97, Springer-Verlag, Berlin-New York, 1977, 1979. MR0500056 (58:17766); MR0540367 (81c:46001)

[10] I. I. Privalov, Boundary properties of analytic functions, GITTL, Moscow-Leningrad, 1950. (Russian) MR0047765(13:926h)

[11] S. V. Kislyakov, On BMO-regular lattices of measurable functions. II, Zap. Nauchn. Sem. S.Peterburg. Otdel. Mat. Inst. Steklov. (POMI) 303 (2003), 161-168; English transl., J. Math. Sci. (N. Y.) 129 (2005), no. 4, 4018-4021. MR2037537 (2004i:46041)

[12] _ On BMO-regular lattices of measurable functions, Algebra i Analiz 14 (2002), no. 2, 117135; English transl., St. Petersburg Math. J. 14 (2003), no. 2, 273-286. MR1925883(2003m:46043)

[13] _ On BMO-regular couples of lattices of measurable functions, Studia Math. 159 (2003), no. 2, 277-290. MR2052223 (2005f:46060)

[14] _ Bourgain analytic projection revisited, Proc. Amer. Math. Soc. 126 (1998), 3307-3314. MR $1458882(99 a: 46039)$

[15] B. S. Mityagin, An interpolation theorem for modular spaces, Mat. Sb. (N. S.) 66 (1965), no. 4, 473-482. (Russian) MR.0177299 (31:1562)

[16] J. Bergh and J. Löfström, Interpolation spaces. An introduction, Grundlehren Math. Wiss., Bd. 223, Springer-Verlag, Berlin-New York, 1976. MR0482275 (58:2349)

[17] K. Hoffman, Banach spaces of analytic functions, Prentice-Hall, Englewood Cliffs, NJ, 1962. MR0133008 (24:A2844)

[18] L. V. Kantorovich and G. P. Akilov, Functional analysis, Nevskiü Dialekt, St. Petersburg, 2004; English transl., Pergamon Press, Oxford-Elmsford, NY, 1982. MR0664597 (83h:46002)

[19] W. Rudin, Function theory in polydiscs, Benjamin, New York-Amsterdam, 1969. MR0255841 (41:501)

[20] N. K. Nikol'skiı̌, Lectures on the shift operator, Nauka, Moscow, 1980; English transl., Treatise on the shift operator. Spectral function theory, Grundlehren Math. Wiss., Bd. 273, Springer-Verlag, Berlin, 1986. MR0575166 (82i:47013), MR0827223 (87i:47042)

[21] L. Diening, P. Harjulehto, P. Hästö, and M. Ruzicka, Lebesgue and Sobolev spaces with variable exponents (a book manuscript in preparation, see http://www.helsinki.fi/ pharjule/varsob/ publications.shtml).

[22] N. J. Kalton, Complex interpolation of Hardy-type subspaces, Math. Nachr. 171 (1995), 227-258. MR $1316360(96 \mathrm{a}: 46055)$

[23] Ky Fan, Fixed-point and minimax theorems in locally convex topological linear spaces, Proc. Nat. Acad. Sci. U.S.A. 38 (1952), 121-126. MR0047317 (13:858d)

[24] G. Ya. Lozanovskiı̌, Certain Banach lattices, Sibirsk. Mat. Zh. 10 (1969), 584-599. (Russian) MR0241949 (39:3285)

[25] D. V. Rutsky, Two remarks on the relationship between BMO-regularity and analytic stability of interpolation for lattices of measurable functions, Zap. Nauchn. Sem. S.-Peterburg. Otdel. Mat. Inst. Steklov. (POMI) 366 (2009), 102-115; English transl., J. Math. Sci. (N.Y.) 165 (2010), no. 4, 483-490. MR22749153

[26] _ Remarks on BMO-regularity and AK-stability, Zap. Nauchn. Sem. S.-Peterburg. Otdel. Mat. Inst. Steklov. (POMI) 376 (2010), 116-166. (Russian) MR2749288 
[27] _ Thesis (C. Sc.), S.-Petersburg. Otdel. Mat. Inst. Steklov (POMI), St. Petersburg, 2011. (Russian)

[28] Y. Benyamini and Y. Sternfeld, Spheres in infinite-dimensional normed spaces are Lipschitz contractible, Proc. Amer. Math. Soc. 88 (1983), no. 3, 439-445. MR0699410 (85h:46028)

[29] D. Cruz-Uribe, A. Fiorenza, J. M. Martell, and C. Pérez, The boundedness of classical operators on variable $L^{p}$ spaces, Ann. Acad. Sci. Fenn. Math. 31 (2006), no. 1, 239-264. MR2210118 (2006m:42029)

[30] J. L. Rubio de Francia, Factorization theory and $A_{p}$ weights, Amer. J. Math. 106 (1984), 533-547. MR0745140 (86a:47028a)

[31] P. Hästö and L. Diening, Muckenhoupt weights in variable exponent spaces, Preprint, see http://www.helsinki.fi/ pharjule/varsob/publications.shtml, 2010.

[32] V. Kokilashvili and V. Paatashvili, On variable Hardy and Smirnov classes of analytic functions, Georgian Internat. J. 1 (2008), no. 2, 181-195.

[33] V. I. Gavrilov and A. V. Subbotin, A maximal version of the multidimensional Khinchin-Ostrovskiน theorem and applications, Dokl. Akad. Nauk 405 (2005), no. 2, 158-160. (Russian) MR2262530 (2007i:32003)

[34] T. Kopaliani, Interpolation theorems for variable exponent Lebesgue spaces, J. Funct. Anal. 257 (2009), no. 11, 3541-3551. MR2572260 (2011b:42063)

[35] Quan Hua Xu, Some properties of the quotient space $L^{1}\left(\mathbb{T}^{n}\right) / H^{1}\left(\mathbb{T}^{n}\right)$, Illinois J. Math. 37 (1993), no. 3, 437-454. MR.1219649 (94k:46049)

[36] _ Notes on interpolation of Hardy spaces, Ann. Inst. Fourier (Grenoble) 42 (1992), 875-889. MR 1196097 (94e:46135a)

[37] D. S. Anisimov and S. V. Kislyakov, Double singular integrals: Interpolation and correction, Algebra i Analiz 16 (2004), no. 5, 1-33; English transl., St. Petersburg Math. J. 16 (2005), no. 5, 749-772. MR2106665 (2005h:42029)

[38] S. V. Kislyakov and Quan Hua Xu, Real interpolation and singular integrals, Algebra i Analiz 8 (1996), no. 4, 75-109; English transl., St. Petersburg Math. J. 8 (1997), no. 4, 593-615. MR1418256 (98c:46161)

[39] A. K. Lerner, Weighted norm inequalities for the local sharp maximal function, J. Fourier Anal. Appl. 10 (2004), no. 5, 465-474. MR2093912 (2005i:42028)

[40] B. Jawerth and A. Torchinsky, Local sharp maximal functions, J. Approx. Theory 43 (1985), 231270. MR0779906 (86k:42034)

[41] J. L. Krivine, Théorèmes de factorisation dans les espaces réticulés, Séminaire Maurey-Schwartz 1973-1974, Exp. No. 22-23, Centre de Math., Ecole Polytech., Paris, 1974. MR0440334 (55:13209)

[42] E. M. Dyn'kin and B. P. Osilenker, Weighted estimates for singular integrals and their applications, Itogi Nauki i Techniki. Ser. Mat. Anal., vol. 21, VINITI, Moscow, 1983, pp. 42-129. (Russian) MR $0736522(85 \mathrm{j}: 42034)$

[43] T. P. Hytönen, The sharp weighted bound for general Calderón-Zygmund operators, Preprint, http://arxiv.org/abs/1007.4330, 2010, jul.

[44] T. P. Hytönen, C. Pérez, S. Treil, and A. Volberg, Sharp weighted estimates for dyadic shifts and the $A_{2}$ conjecture, Preprint, http://arxiv.org/abs/1010.0755v2, 2010, dec.

[45] J. Orobitg and C. Pérez, $A_{p}$ weights for nondoubling measures in $R^{n}$ and applications, Trans. Amer. Math. Soc. 354 (2002), 2013-2033. MR.1881028 (2002k:42044)

[46] J. L. Rubio de Francia, Linear operators in Banach lattices and weighted $L^{2}$-inequalities, Math. Nachr. 133 (1987), 197-209. MR0912429 (89d:47070)

St. Petersburg Branch, Steklov Mathematical Institute, Fontanka 27, St. Petersburg 191023, RUSSIA

E-mail address: rutsky@pdmi.ras.ru

Received 21/OCT/2010

Translated by THE AUTHOR 